\title{
Effectiveness of the displacement-based seismic performance assessment for continuous RC bridges and proposed extensions
}

\author{
Roberto Gentile ${ }^{\mathrm{a}}$, Andrea Nettis ${ }^{\mathrm{b}, *}$, Domenico Raffaele ${ }^{\mathrm{b}}$ \\ ${ }^{a}$ Department of Civil, Environmental and Geomatic Engineering \& Institute for Risk and Disaster Reduction. University College London, \\ London, United Kingdom \\ ${ }^{b}$ Department of Civil, Environmental, Land, Building Engineering and Chemistry. Polytechnic University of Bari, Italy
}

\begin{abstract}
Seismic performance assessment of existing bridges, especially those designed without seismic criteria, is paramount in earthquake-prone countries. To perform this task, the Displacement-Based Assessment (DBA) represents a satisfactory trade-off between simplicity and accuracy. After describing the modal analysis-based DBA procedure, a static-based alternative is proposed in this paper, considering its strengths and limitations. Moreover, an extension of the procedure is proposed to derive the force-displacement curve of the bridge (pseudo-pushover capacity curve). The effectiveness of the DBA methodology, implemented via simplified mechanical models, is discussed through parametric analyses to address its practical applications. The DBA approach, both modal and static, is herein adopted for the transverse analysis of a set of 36 reinforced concrete continuous-deck bridges up to six spans, with pier height in the range 8-20m and two different values of the deck transverse stiffness. Additional sensitivity analyses (24 case studies) are conducted to investigate the accuracy of the two approaches considering: 1) the length of the bridge, 2) the amount of longitudinal reinforcement in the piers; 3) different pier typologies. The results are compared to numerical pushover and time-history analyses using three suites of 10 scaled, natural ground motions respectively consistent with low-, medium- and high-seismicity sites. For the majority of the case studies, the resulting performance assessments fall within one standard deviation of the results of the time-history analyses. For this reason, the displacement-based modal or static approaches can represent a valid alternative to numerical non-linear static analyses for continuous bridges with six spans or less.
\end{abstract}

Keywords: Displacement-based seismic assessment, RC bridges, capacity/demand ratio, capacity spectrum method, pushover analyses, non-linear time-history analyses.

\section{Introduction and motivation}

In high earthquake risk countries, the seismic assessment of bridges is particularly important since often such structures represent crucial nodes of the transportation networks and have strategic functions. This is demonstrated by field observations in the aftermath of major earthquakes, e.g. the $M_{w}=7.8,2016$ Kaikoura earthquake [1]. Moreover, in many countries (e.g. Italy), many of the existing bridges are designed with non-seismic methods, rather than advanced techniques (e.g. [2]), thus increasing the need for an evaluation of their seismic behaviour.

Non-linear time-history analysis (NLTHA) is arguably the most refined available tool to perform a seismic performance assessment, provided that significant computational effort is spent, and time/skills are available to setup the numerical model of the bridge and interpret its results. Similar difficulties can arise adopting advanced non-linear static procedures (NSPs), such as the adaptive pushover. Based on previous work [3, 4], force-based and displacement-based adaptive pushover (FAP and DAP) techniques were proposed $[5,6]$. These represent improvements of the conventional invariant pushover since they allow to account for the effect that progressive stiffness degradation might have on the distribution

\footnotetext{
* Corresponding author

Email addresses: r.gentile@ucl.ac.uk (Roberto Gentile), andrea.nettis@poliba.it (Andrea Nettis), domenico.raffaele@poliba.it (Domenico Raffaele)
} 
of seismic forces. Both the FAP and DAP were tested on reinforced concrete (RC) bridges proving high accuracy [7, 8].

Part of the above-mentioned difficulties in non-linear numerical models are overcome using the Displacement-Based Assessment (DBA), which represents a satisfactory trade-off between simplicity of the analysis and accuracy of the results. Such features render DBA also suitable for the analysis of large portfolios, possibly coupled with simplified methods for the characterisation of structural members (e.g. Cosenza et al., 2011 [9], Gentile et al., 2018a,b [10, 11] for RC members).

The DBA derives from its design counterpart (Priestley et al., 2007 [12]), which was firstly proposed for bridges by Kowalsky et al. 2002 [13]. Extensions to the procedure that include the effects of higher modes in the design have also been proposed $[14,15]$. The DBA procedure for bridges was proposed by Sadan et al. 2013 [16] for continuous-deck configurations with pinned abutments, and involves the modal analysis of the bridge based on secant stiffness properties for the piers (effective modal analysis [13]). The DBA was extended by Ni et al., 2014 [17] to include soil structure interaction. Further work by Cardone (2014) [8] aimed at proposing performance displacement profiles correspondent to a limit state in various members of the bridge (piers, abutments, joints, bearing devices and shear keys). In such cases, the full capacity curve of the bridge is not derived.

After describing the existing DBA procedure based on modal analysis, this paper proposes an alternative procedure based on a static analysis. Moreover, it is proposed an extension of the DBA procedures, both modal and static, to calculate the full pseudo-pushover capacity curve of bridges. Finally, an open-access application is provided for the above calculations. Since a relatively less-complex model and analysis type are used, the static-based DBA is deemed to be easier in the practical applications, for example allowing to use an electronic spreadsheet (possibly validated against the provided application) instead of a numerical computer model. Using a static rather than modal analysis is an attempt to render the procedure "practice oriented" as much as possible, aiming at the adoption of simple mechanical models constructed by the user, while building refined numerical ones only for the final validation (and vice versa). This could potentially reduce (or avoid) the "black-box" effect due to the adoption of highly-refined models.

The above-mentioned modal and static approaches are based on the repeated application of the DBA for increasing values of the displacement profile, and are herein called Displacement-Based Pseudo Pushover (DBPP). The concept is similar to what done for RC buildings by proposing the Simple Lateral Mechanism Analysis (SLaMA) method [18, 19, 20, 21, 22]. The DBPP allows obtaining a pseudo-pushover curve of the bridge, for which the term "pseudo" refers to a series of linear analyses using secant rather than tangent stiffness properties for the members [18]. Using such curve within a capacity spectrum-based assessment approach, allows calculating a variety of engineering demand parameters (for one or more spectra). This also allows performing fragility analysis and risk assessment.

The scope of this paper is limited to the transverse analysis of continuous deck bridges. The longitudinal analysis of such bridges is deemed to be a less-complex application of the proposed solution, since it is based on a much simpler static scheme (piers in parallel which are forced to the same top displacement). Moreover, multi-span simply-supported bridges are not considered since in this case each span can be modelled as a Single Degree of Freedom (SDoF) system, both in the transverse and longitudinal directions. Finally, although the main case-study dataset (Section 3.1) refers to RC continuous-deck bridges with single-column piers, straightforward modifications in characterising the simplified structural model allow to consider other deck typologies, pier-to-deck connections, and pier typologies (some of which are herein considered in an additional sensitivity analysis).

The effectiveness of the DBPP approach is analysed by means of the application to a dataset composed of 36 RC bridge case studies with continuous deck, comprising wide ranges for the number of spans (2-6), the height distribution of the piers $(8-20 \mathrm{~m})$, the force-displacement response of the piers and the moment of inertia of the deck (flexure in the transverse direction of the bridge). Firstly, the results are compared with refined numerically-based pushover analyses using two different force profiles. The Capacity Spectrum Method (CSM, Freeman et al., 1998 [23]) is applied using such curves to derive a performance point. The results are critically compared with the average of time-history analyses using 
three suites of 10 scaled, natural ground motions respectively consistent with low-, medium- and high-seismicity sites. Finally, additional sensitivity analyses are conducted to investigate the accuracy of both analytical approaches in relation to the bridge length, the amount of longitudinal reinforcement in the piers and different pier typologies. On the basis of the obtained outcomes, some applicability limits are suggested to address a practical and appropriate use of these DBA methodologies.

\section{Description of the DBA for bridges and proposed extensions}

The DBA procedure for bridges aims at the identification of their displacement capacity (for a given limit state) expressed in terms of equivalent SDoF properties. The assessment is performed comparing the displacement capacity and demand at the corresponding limit state, which depends on effective structural period and equivalent viscous damping of the SDoF system.

An initial knowledge phase is required to define the geometry and detailing of the investigated bridge. Subsequently, limit displacements (or drifts) should be defined for each member composing the bridge (e.g. piers, abutments, bearings). The DBA allows to identify the displacement profile, and related base shear, associated with the limit displacement/drift for one or more members in the bridge.

\subsection{Overview of the DBA procedure based on modal analysis}

The DBA procedure based on modal analysis [16] is described herein. Firstly, the force-displacement characterisation of the piers and abutments should be provided. While an elastic behaviour is often appropriate for the abutments, the non-linear force-displacement curve of the piers is needed. For RC single-column piers this can be calculated based on equivalent cantilever models [2] which take into account the mass distribution along the pier height (including the pier cap and a portion of the deck). The model choice (with particular reference to the shear span of the pier) can change depending on the boundary conditions at the deck connection [2]. The height $H$ of the equivalent cantilever can be calculated according to Eq. 1 , where $H_{p}$ and $H_{d}$ are respectively the height of the pier and the deck centre of mass. The equivalent cantilever mass $m$ involves the mass of pier $m_{p}$, the pier cap $m_{p c}$ and the deck portion between two mid-spans $m_{d}$. Alternatively, the height of the equivalent cantilever can be set equal to the height of the deck centre of mass. If a high degree of deck torsional restraint is present, a different formulation [12] of the equivalent cantilever height should be chosen.

$$
H=\frac{\left(m_{p c}+0.3 m_{p}\right) H_{p}+m_{d} H_{d}}{m} \quad \text { where } \quad m=0.3 m_{p}+m_{p c}+m_{d}
$$

The force-displacement characterisation of each equivalent cantilever requires the (bi-linear) moment-curvature relationship for the base section of the pier. This can be calculated with simplified formulations [10, 11] or numerical approaches such as the computer code CUMBIA [24]. The force-displacement curve can be calculated with Equations 2 to 7 (Priestley et al., 2007 [12]), in which $\varphi_{Y}$ and $\varphi_{U}$ are the yielding and ultimate curvatures, $M_{Y}$ and $M_{U}$ are the yielding and ultimate moments, $\Delta_{Y}$ and $\Delta_{U}$ are the yielding and ultimate displacements. $L_{S P}$ is the strain penetration length, $f_{y}$ and $f_{u}$ are the yielding and ultimate steel stresses and $d_{b l}$ is the mean bar diameter in the pier. It is worth mentioning that alternative failure modes should be considered, such as lap-splice [2] or buckling [25] of the longitudinal bars or shear failure [26]. If one or more alternative failure modes anticipates (or prevents) the flexural hinging, the force-displacement relationship of the piers should be modified in the pre-processing phase, before using it in the analysis.

$$
F_{Y / U}=\frac{M_{Y / U}}{H+L_{S P}}
$$




$$
\begin{gathered}
\Delta_{Y}=\frac{\varphi_{Y}\left(H+L_{S P}\right)^{2}}{3} \\
\Delta_{U}=\Delta_{Y}+\Delta_{P}=\Delta_{Y}+\left(\varphi_{Y}-\varphi_{U}\right) L_{P} H \\
L_{S P}=0.022 f_{y} d_{b l} \\
L_{P}=k H+L_{S P} \\
k=0.2\left(\frac{f_{u}}{f_{y}}-1\right) \leq 0.08
\end{gathered}
$$

The DBA procedure based on modal analysis (Figure 1) starts with the individuation of the member that controls the considered limit state, which in turn allows to select a "control node" in the structural model of the bridge, and to set its displacement $\left(\Delta_{c}\right)$. To this aim, the so-called structural component modelling approach by Priestley et al., 1996 [2] is used. For analyses in the transverse direction, such scheme (Figure 1) is composed by an elastic beam (the deck) on spring supports (piers and abutments). In the longitudinal direction, the bridge can be modelled considering springs in parallel (piers and abutments) which are forced to the same displacement. Each pier in multi-span simplysupported bridges can be modelled as individual SDoF systems [2] (one per each pier), which are calibrated according to their tributary mass. Finally, the behaviour of other structural members (e.g. bearings, shear keys, abutment backfill) should be considered in the pre-processing phase of the analysis. In particular, each non-linear spring member should be considered as a combination (in series or in parallel) of two or more members (e.g. pier + bearing + shear key). The resulting force-displacement curve should be used in the analysis. For brevity, such situation is not explicitly considered in the case-study dataset of this paper.

An initial guess of the displacement shape is scaled in such a way that the displacement of the control node is equal to $\Delta_{c}$. This allows to calculate the displacement shape $\Delta_{i}$. The shear force in each pier or abutment $\left(V_{i}\right)$ is derived using the appropriate force-displacement curve, and the secant stiffness $\left(k_{i}\right)$ is calculated according to Eq. 8 . A first modal (eigenvalue) analysis is carried out, deriving the first transverse modal shape $\left(\phi_{i}\right)$ and its participating mass $\left(M_{1}^{*}\right)$. The first modal shape is scaled according to Eq. 9 to derive a new displacement profile $\left(\Delta_{i}^{\prime}\right)$, which is compared to the previous guess $\left(\Delta_{i}\right)$. The secant stiffness is updated (Eq. 8) and a new eigenvalue analysis is performed until the calculated displacement profile stabilises. It is worth mentioning that, using a reasonable value of the tolerance (e.g. 0.001), three or four iterations are usually sufficient.

$$
\begin{gathered}
k_{i}=\frac{V_{i}}{\Delta_{i}} \\
\Delta_{i}^{\prime}=\Delta_{c} \frac{\phi_{i}}{\phi_{c}}
\end{gathered}
$$

If the participating mass of the first vibration mode is less than a given threshold (e.g. 70\%), the effect of higher modes should be taken into account in each iteration. This can be done calculating the Effective Mode Shape (EMS [13]), considering a number of vibration modes such that the cumulative participating mass is equal or greater than $90 \%$. The performance displacement for each considered mode $j$ is calculated according to Eq. 10, where $\Gamma_{j}$ and $T_{j}$ are the modal participation factor and the period of vibration of mode $j$. Moreover, $S_{d}\left(T_{j}\right)$ is the displacement demand calculated with an hazard-specific 5\% damped elastic spectrum. The final displacement profile $\Delta_{i}$ is finally obtained with a modal 


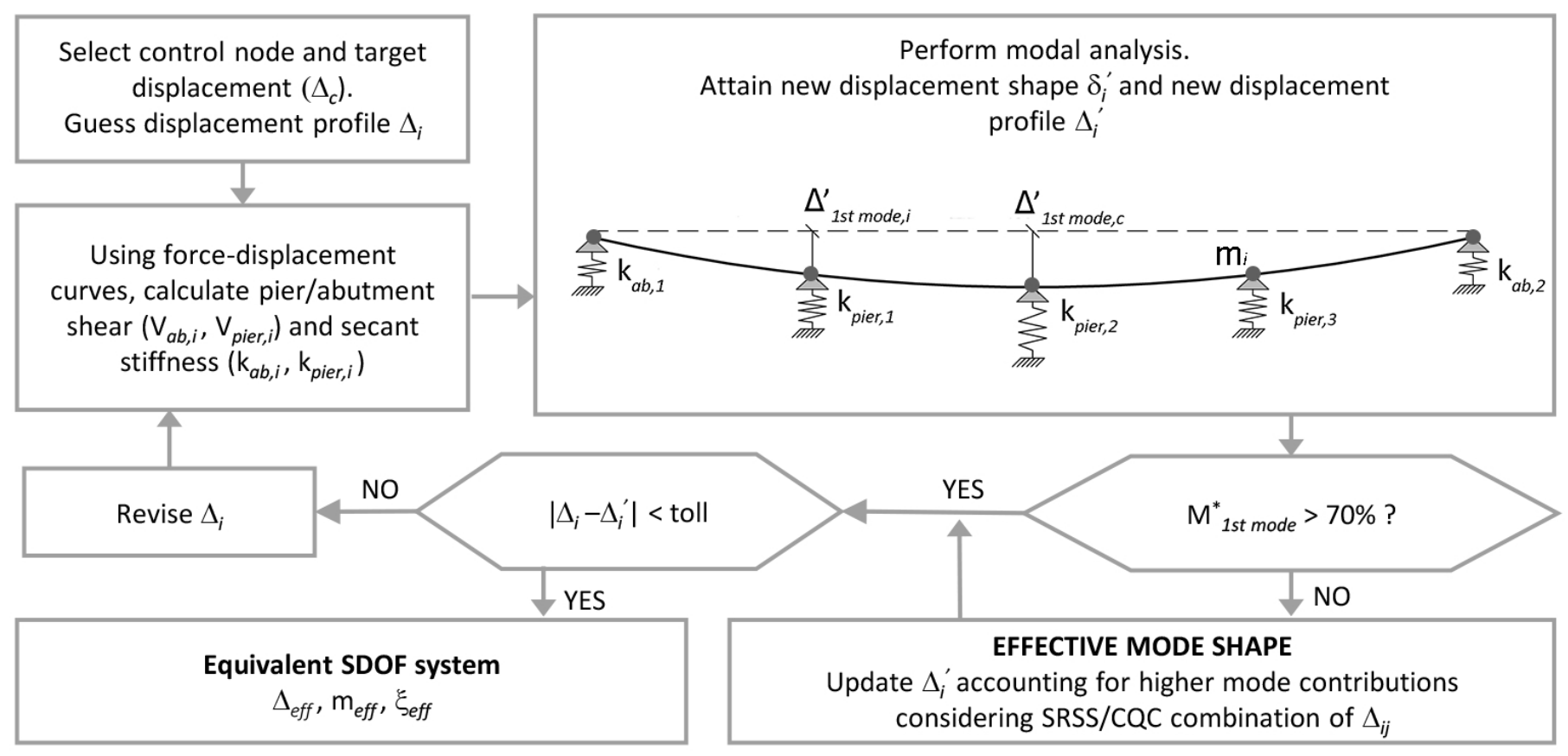

Figure 1: DBA procedure based on modal analysis.

combination method such as the Square Root of the Sum of the Squares (SRSS) or the Complete Quadratic Combination $(\mathrm{CQC})$.

$$
\Delta_{i, j}=\phi_{i, j} \Gamma_{j} S_{d}\left(T_{j}\right)
$$

The final step of the procedure is the characterisation of the equivalent (effective) SDoF system which represents the bridge. This is done consistently with the approach by Priestley et al. [12]. The effective displacement $\left(\Delta_{e f f}\right)$ and effective mass $\left(m_{e f f}\right)$ are computed according to Equations 11 and 12. The effective damping of the system is calculated accounting for the contribution of all the bridge members, including their elastic and possible hysteretic contributions. Equations 13 and 14 allow to calculate it considering 5\% elastic damping for the abutments and $2 \%$ for the deck. In such equations, $\Delta_{a b}$ and $V_{a b}$ are the displacement and shear of one abutment, $\Delta_{p i e r, k}$ and $V_{p i e r, k}$ are the displacement and shear of the $k^{t h}$ pier and $\mu_{k}=\Delta_{\text {pier }, k} / \Delta_{Y \text { pier }, k}$. The seismic assessment can be performed comparing the displacement capacity of the SDoF with the displacement demand calculated on an hazard-compatible displacement spectrum.

$$
\begin{aligned}
& \Delta_{e f f}=\frac{\sum m_{i} \Delta_{i}^{2}}{\sum m_{i} \Delta_{i}} \\
& m_{e f f}=\frac{\sum m_{i} \Delta_{i}}{\Delta_{e f f}} \\
& \xi_{e f f}=\frac{0.05 \Delta_{a b, 1} V_{a b, 1}+0.05 \Delta_{a b, 2} V_{a b, 2}+0.02 \Delta_{e f f}\left(V_{a b, 1}+V_{a b, 2}\right)+\sum \xi_{p i e r, k} \Delta_{p i e r, k} V_{p i e r, k}}{\Delta_{a b, 1} V_{a b, 1}+\Delta_{a b, 2} V_{a b, 2}+\Delta_{e f f}\left(V_{a b, 1}+V_{a b, 2}\right)+\sum \Delta_{p i e r, k} V_{p i e r, k}} \\
& \xi_{\text {pier }, k}=0.05+\frac{0.444\left(\mu_{k}-1\right)}{\mu_{k} \pi}
\end{aligned}
$$

\subsection{Proposed alternative DBA procedure based on static analysis}

To provide a more practice-oriented tool, an alternative DBA procedure is proposed which is based on static analysis. It starts with the definition of a control node, which is related to the member that controls the considered limit state. A guess of the displacement shape is defined and it is scaled in such a way that the displacement of the control node is equal 


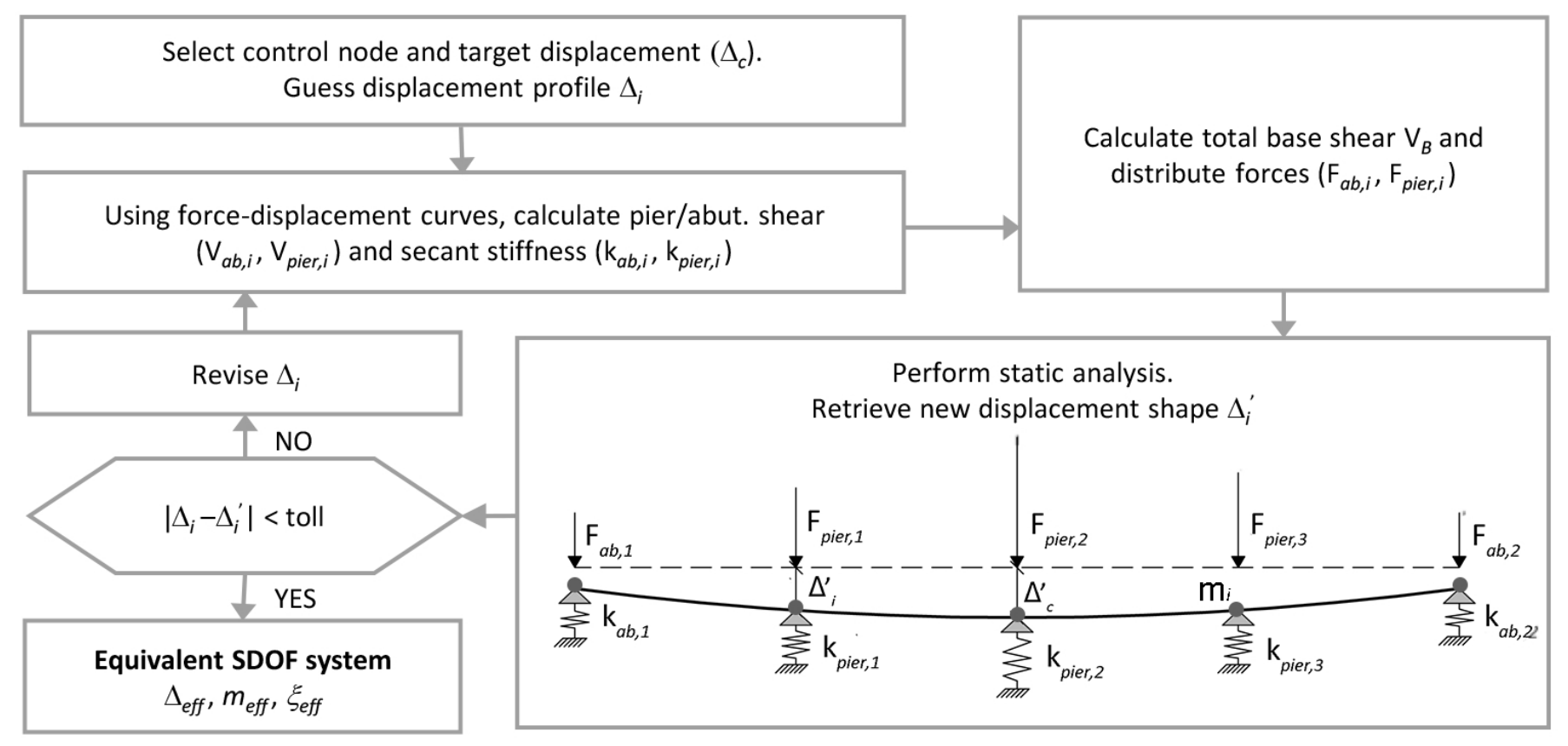

Figure 2: Proposed alternative DBA procedure based on static analysis.

to $\Delta_{c}$. The displacement of each member $\left(\Delta_{i}\right)$ is used to interpolate the force-displacement curve of the sub-structure members and derive the shear $\left(V_{i}\right)$ and, in turn, the secant stiffness $\left(k_{i}\right)$.

The distribution of inertia forces is calculated with Eq. 15, where $V_{B}$ is the base shear. A static analysis is performed, and the calculated displacement profile $\left(\Delta_{i}^{\prime}\right)$ is compared with the initial guess to check for convergence. A new static analysis is conducted, after updating all the involved parameters, until the displacement profile stabilises. The characterisation of the SDoF system and the final check against the seismic demand are performed according to the same steps described in Section 2.1 .

The displacement profiles by Priestley et al. [12] or Cardone [8] could be adopted to select the initial guess. Nevertheless, a sensitivity analysis in this work demonstrated that the initial guess does not affect the achievement of the convergence for the case studies herein analysed. In fact, regardless of the initial guess, three of four iterations of the analysis are normally sufficient if a tolerance of $1 \mathrm{~mm}$ is adopted. In the specific case of this work, a parabolic displacement pattern is assumed as initial guess for all the analysed bridges.

$$
F_{i}=\frac{m_{i} \Delta_{i}}{\sum m_{i} \Delta_{i}} V_{B}
$$

\subsection{Analytical displacement-based pseudo pushover}

Both the modal (Section 2.1) and the static (Section 2.2) DBA procedures are conceptually simple and they can be performed using electronic worksheets or code routines, using analytical static schemes rather than finite element numerical models. Therefore, with the aim of fully exploiting the potentiality of the DBA approaches, it is proposed to extend them to derive the full capacity curve of the bridge. Such process is herein referred as displacement-based pseudo pushover (DBPP). An open access, stand-alone application is provided for these calculations [27]. The basic idea is to repeat the modal or static DBA procedure for increasing displacements, allowing to have a thorough information on the behaviour of the analysed bridge with a particularly small increase in computational cost. This allows to calculate, for increasing displacements, the properties of the SDoF system $\left(\Delta_{e f f}, m_{e f f}, \xi_{e f f}\right)$ and the Engineering Demand Parameters (EDP) for each single member (displacements of the deck, shear in the abutments, shear and base moment of the piers, etc.). Finally, it is possible to plot the base shear vs effective displacement curve. 
The capacity curve deriving from this process is based on series of linear analyses (modal or static) of a system whose members are characterised by the secant stiffness compatible with increasing levels of displacement. Such idea is similar to the (more familiar) concept of a pseudo pushover analysis, which refers to a series of linear elastic analyses of a computer model based on secant stiffness. Such approach, also allowed in international seismic guidelines [18], theoretically allows to achieve the same results of a standard pushover analysis.

Similarly to a numerical analysis, the number of needed steps (or the size of each step) depends on the "smoothness" of the capacity curve required by the user and on the adopted force-displacement relationships of the members (e.g. piers, abutments, bearings, shear keys). If the member capacity curves are multi-linear, $\sum_{i=1}^{m} b_{i}$ analysis steps are needed, where $m$ is the number of non-linear members and $b_{i}$ is the number of linear branches of the capacity curve of member $i$. In such case, guidance by Cardone (2014) [8] can be adopted to define the displacement profiles for each analysis step. If the process is implemented in a routine or electronic spreadsheet, it could be less-demanding to run the analysis for equallyspaced displacement increments (using a sufficiently-small step size) and to derive the relevant limit state displacement profiles by post-processing the results. On the other hand, if smooth capacity curves are adopted for the members (e.g. fibre-based force-displacement analysis for the piers), the latter approach is suggested using a relatively-small step size (e.g. $1 \mathrm{~cm})$.

From the practical point of view, the DBPP starts with the (arbitrary) definition of a control node in the static scheme of the bridge. It is suggested to select the top of a relatively-central pier as control node, although the resulting capacity curve is independent from such choice. The displacement of the control node is set and the modal (Figure 1) or static (Figure 2) DBA is carried out. The process is repeated for an arbitrary number of times, checking for each step if one or more members in the system have exceeded their displacement capacity. By definition, each analysed step refers to an independent displacement profile and equivalent SDoF system which are compatible with the related secant stiffness, i.e. the procedure is "adaptive". In the context of the DBPPm, if the EMS is needed in the elastic range $\left(M_{1}^{*}<70 \%\right)$, it should be carried out in the non-linear range regardless of the participating mass of the effective first mode (i.e. even if $\left.M_{1}^{*}>70 \%\right)$.

Finally, once the DBPP curve is obtained, the seismic assessment evaluation can be performed with an AccelerationDisplacement Response Spectrum (ADRS) method. As an example, the CSM [23] can be applied using the properties $\left(m_{\text {eff }}\right.$ and $\left.\xi_{\text {eff }}\right)$ of the SDoF defined for each step, as also proposed by Casarotti and Pinho [28]. The defined Performance Point (PP) is compared with the displacement capacity of the system to perform the final assessment.

Apart from the intrinsic simplicity of the method, the adoption of the DB non-linear curves allows the possibility to investigate the behaviour of the bridge in a "what if" fashion, which can be particularly useful for the design of retrofit options. In other words, by individuating the response of the structure in correspondence of successive performance limits of the members, it is possible to take into account the redundancy of the bridge and/or design redundant retrofit strategies.

\section{Assumptions for the parametric analysis}

\subsection{Description of the case study bridges}

The dataset of case studies for this parametric analysis is composed of $36 \mathrm{RC}$, continuous-deck, straight bridges with pinned deck-pier and deck-abutments connections (Figure 3). Two deck typologies are considered. The first (J50) has a moment of inertia (for transverse flexure) equal to $52.5 \mathrm{~m}^{4}$ and is composed of three $\mathrm{V}$-shaped $1.8 \mathrm{~m}$-high pre-cast beams and a $11.5 m$-wide slab (0.3m-deep). For the second deck typology (J100), the moment of inertia is equal to $104.1 m^{4}$, the number of $1.8 \mathrm{~m}$-high beams is equal to four and the slab width is equal to $14 \mathrm{~m}$. The dimensions of the pier caps related to each deck typology are indicated in Figure 3. 
For each deck typology, 18 different bridge geometries are considered, with two, four or six 35m-long spans. Pier heights of $8 \mathrm{~m}, 15 \mathrm{~m}$ and $20 \mathrm{~m}$ are adopted to define regular and irregular geometric configurations (Figure 3). As an example, the case study labelled as $B 132$ is a four-span bridge with a $8 m$-, $20 m$ - and $15 m$-high piers. The pier cross section, assumed to be equal for all the piers, is circular with $3 m$ diameter. The longitudinal reinforcement is composed of $63 \phi 26$ bars (with ratio $\rho_{l}=0.47 \%$ ) while the transverse reinforcement is composed of $100 \mathrm{~mm}$-spaced $\phi 10$ bars (with volumetric ratio $\rho_{t}=0.1 \%$ ). The shear strength [26] of the piers is considerably higher than the corresponding flexural one, even for the shortest pier.

The considered unconfined concrete strength is $f_{c}=20 \mathrm{MPa}$ while the steel yield stress is $f_{y}=450 \mathrm{MPa}$. Both the seismic masses and gravity loads (seismic load combination) are calculated based on a concrete density equal to $25 k N / m^{3}$ and a uniform deck load equal to $185 \mathrm{kN} / \mathrm{m}(230 \mathrm{kN} / \mathrm{m}$ for the $\mathrm{J} 100 \mathrm{sub}$-set $)$ to consider both self weight and superimposed loads.

The elastic dynamic behaviour of the selected bridges is dominated by the first mode. Indeed, the first mode participating mass ranges between $74 \%$ and $84 \%$. The elastic (secant-to-yielding) first mode period of the bridges (shown in Figure 5) is observed in the range [0.25s, 1.45s]. On the other hand, the first mode period of the bridges calculated adopting the secant-to-performance point stiffness (CSM-based) falls within the range [0.25s, 1.63s] (also shown in Figure $5)$.

The regularity of the case-study bridges is measured with the relative stiffness index [29] (RS, Eq. 16), which quantifies - with a degree of approximation - the ratio between the (elastic) stiffness of the super-structure and the sub-structure. In such equation, $E J_{d e c k}$ and $L_{\text {deck }}$ are the transverse flexural stiffness and the total length of the deck, respectively. Greater values of RS indicate high regularity of the bridge and low importance of higher modes [15]. The RS is calculated using both the elastic, $R S_{e}$, and secant stiffness, $R S_{p p}$, of the piers (at the CSM-based performance point) to investigate the regularity of the response for increased seismic intensity.

$$
R S=\frac{\frac{384 E J_{\text {deck }}}{5 L_{\text {deck }}}}{\sum_{i} k_{\text {pier }, i}}
$$

\subsection{Assumptions for analytical and numerical analyses}

Both non-linear static procedures (NSPs) and time-history analyses (NLTHA) are performed in this study. The considered NSPs include: static and modal displacement-based pseudo pushover (DBPPs and DBPPm); numerical pushover analysis considering an invariant force profile proportional to the first vibration mode (PUSHm); uniform force profile pushover (PUSHu).

For both the analytical and numerical analyses, the bi-linear moment-curvature relationship of the base section of the piers is adopted. This is calculated using the software CUMBIA [24]. Moment-curvature is carried out adopting the model by Mander et al., 1988 [30] for confined concrete, the model by King et al., 1986 [31] for the steel reinforcement and considering the gravity axial load on the piers. It is worth mentioning that the upper bounds for concrete and steel ultimate strains are set to 0.02 and 0.06, respectively [18]. No strength degradation is considered, since the aim of this work is the analysis of the effectiveness of analytical procedures, rather than capturing strength degradation effects.

The DBPPs and DBPPm procedures are implemented in an ad-hoc Matlab [32] script, allowing for simple and fast calculations. To this aim, the so-called structural component modelling approach by Priestley, 1996 [2] is used, considering the transverse response only. In such analytical model (Figure 1), the continuous deck is modelled as an elastic beam while the piers and the (pinned) abutments are represented by elastic springs. The springs referring to the piers are characterised by a force-displacement curve. This is based on the calculated moment-curvature and adopting Equations 2 to 7 , in which the strain penetration length is neglected. Seismic masses are lumped in the main nodes of the deck, summing the tributary deck mass, the mass of the pier cap and one-third of the pier. 

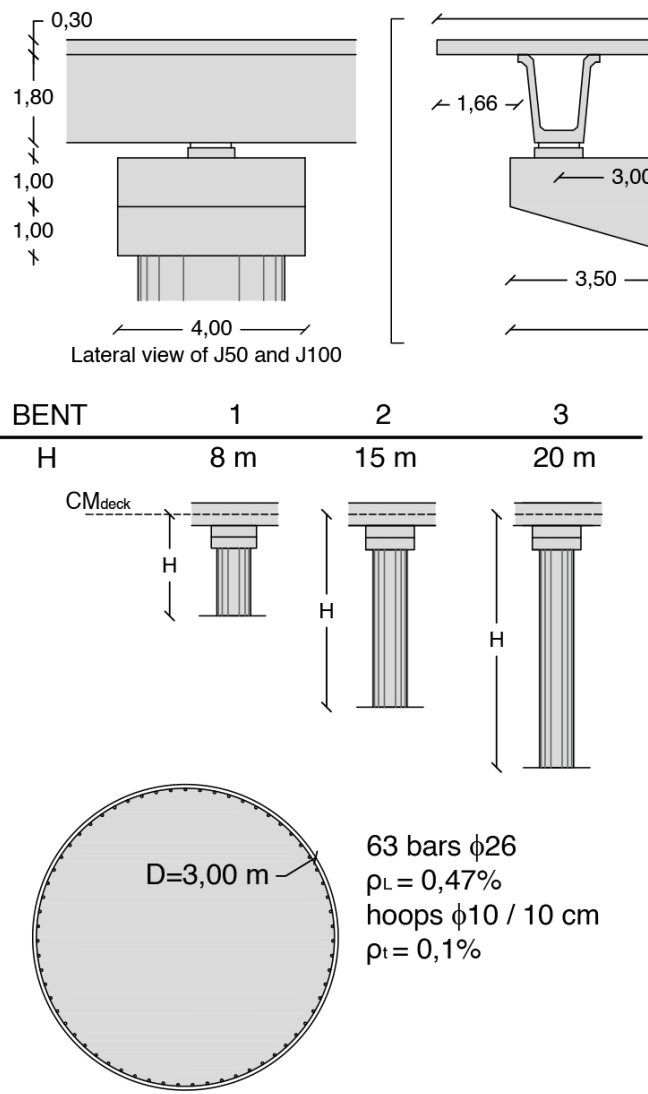

63 bars $\phi 26$

$\rho \mathrm{L}=0,47 \%$

hoops $\phi 10 / 10 \mathrm{~cm}$ $\rho_{t}=0,1 \%$
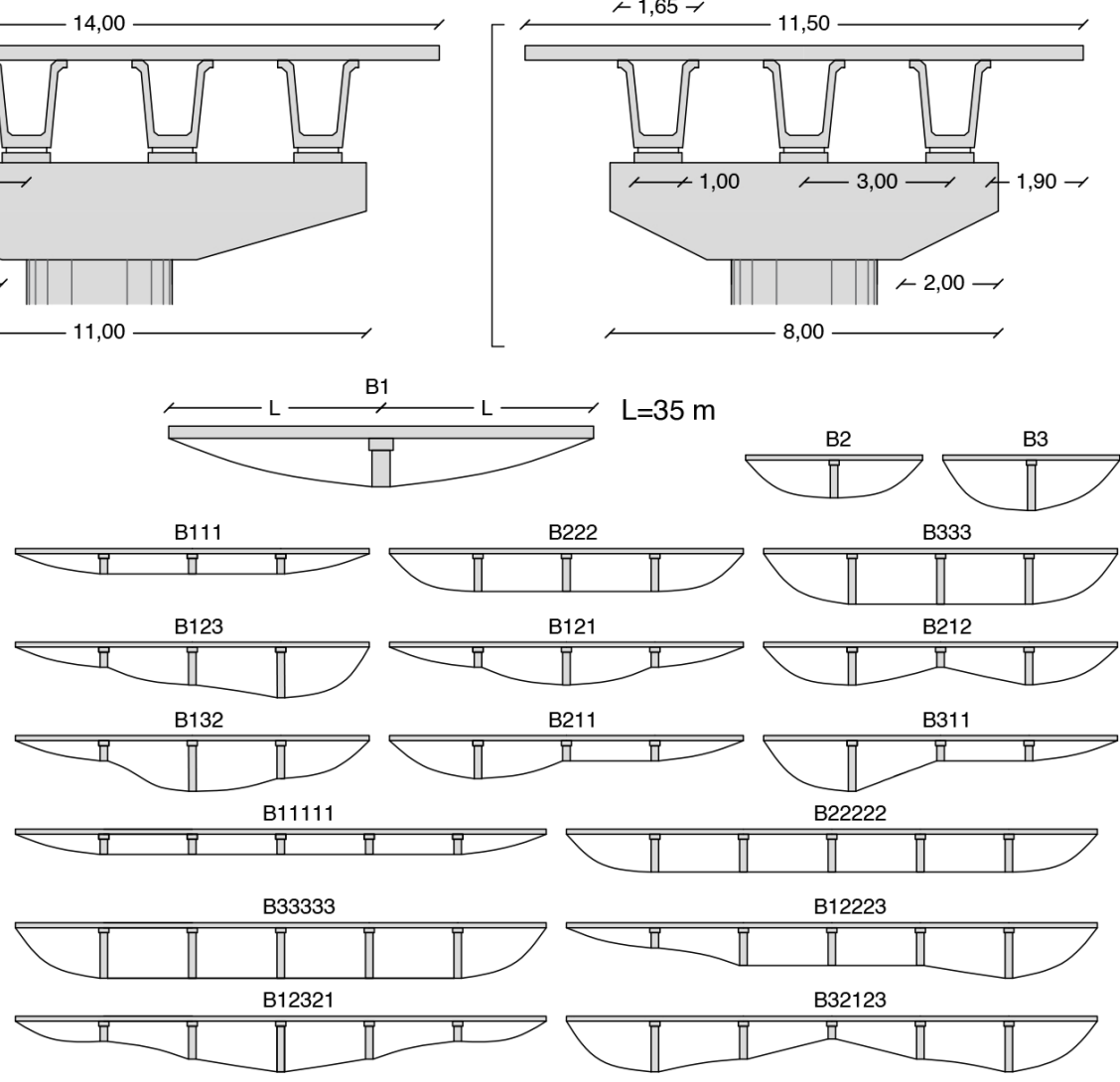

Figure 3: Geometrical configuration of the 36 selected case studies. $\rho_{l}, \rho_{t}$ : longitudinal and transverse reinforcement ratios.

The pushover and time-history numerical analyses are performed using the non-linear finite element software Ruaumoko 3D [33]. The modelling strategy (Figure 4) is based on a lumped plasticity approach in which the deck is an elastic frame member based on uncracked stiffness. In correspondence of each pier, the deck node is part of a body constraint along with mass-less nodes for each girder. Those are connected to the elastic pier cap member through a rigid link and elastic springs representing bearings. Among those, one is modelled as a pinned connection while the others are sliders. A similar approach is adopted for the abutments. Piers are modelled by means of mono-dimensional Giberson elements [34]. The non-linear behaviour of the (fully-fixed) base section of the piers is set consistently with their bi-linear moment-curvature relationship. The cyclic response of the section is modelled with the revised Takeda model [35], using 0.5 and 0 for the unloading and reloading stiffness factors, respectively. The deck mass is distributed on five nodes for each span. The mass of the piers is assigned to four nodes along their height, while three nodes are adopted for pier caps. In the pushover analyses (PUSHm and PUSHu), each node assigned with a mass is part of the lateral load pattern. P- $\Delta$ effects are considered in both the displacement-control pushover and time-history analyses. For the NLTHA, a tangent stiffness proportional damping is selected as suggested by Priestley et al. [12] and a constant $5 \%$ damping is assigned to all the principal modes of the bridges.

\subsection{Seismic demand}

Three ground-motion Intensity Measures (IM) are considered in this study, referring to low-, medium- and highseismicity zones (Calcata, Montesilvano and Cosenza, Italy). Figure 5 shows the related 5\%-damped acceleration response spectra provided by the Italian code [36], related to a return period equal to 450 years, soil type $\mathrm{C}$ (shear wave velocity $V_{s, 30}=180-360 \mathrm{~m} / \mathrm{s}$ ) and an importance factor equal to 1.5. The Peak Ground Acceleration (PGA) is respectively equal to $0.25 \mathrm{~g}, 0.32 \mathrm{~g}$ and $0.42 \mathrm{~g}$. Such spectra are adopted to apply the CSM for both the DBPP curves and the numerical 


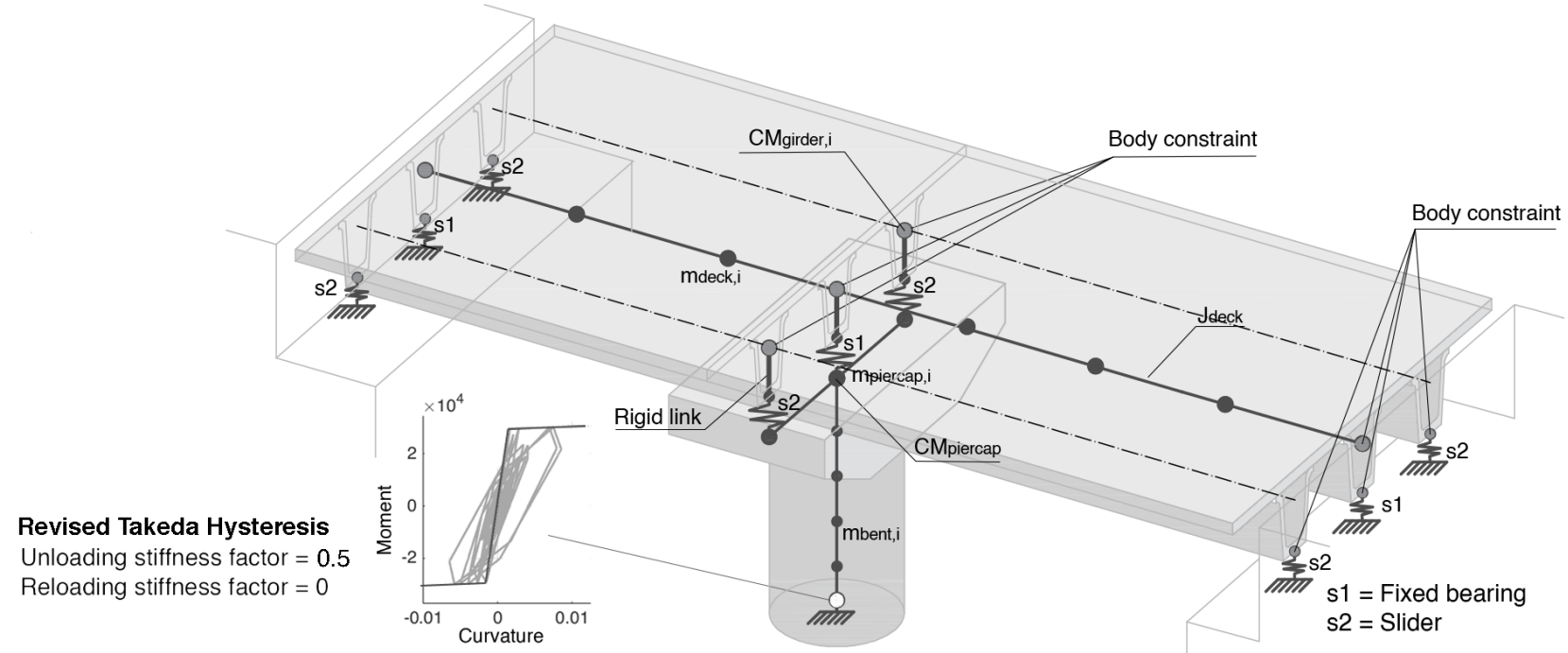

Figure 4: Adopted modelling strategy for numerical time-history and pushover analyses.
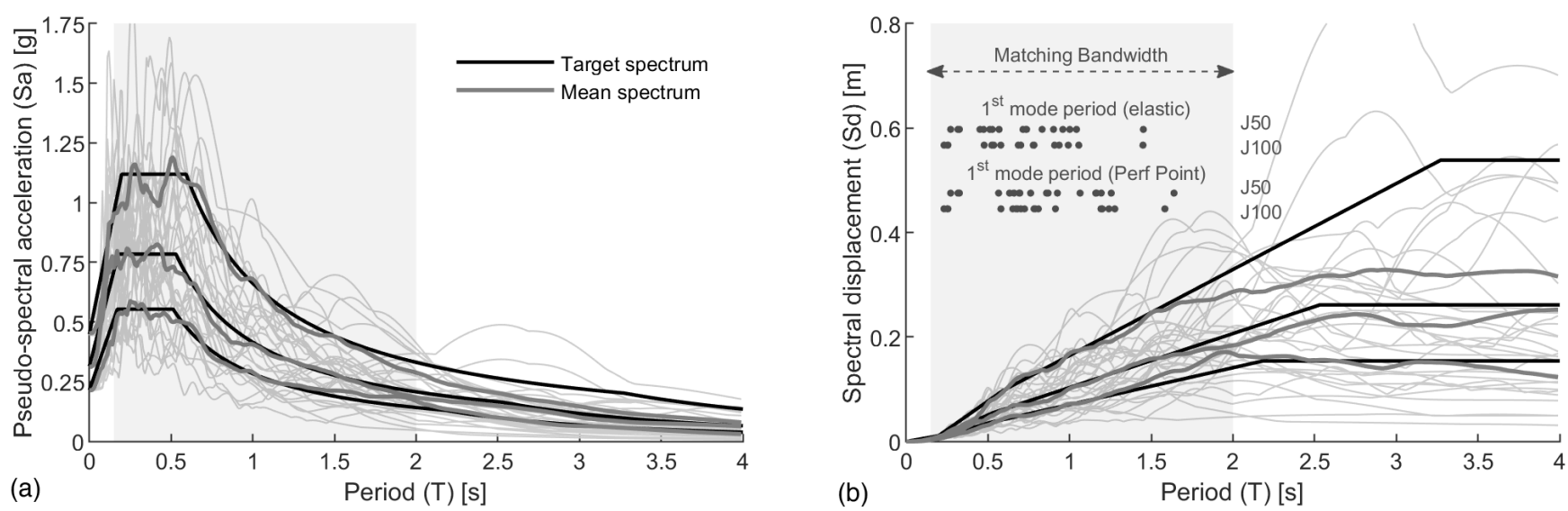

Figure 5: Elastic acceleration (a) and displacement (b) spectra of the scaled selected ground motions.

pushover ones. The equivalent SDoF conversion related to the numerical pushover curves is applied as per the DBA procedures (Section 2.1), for consistency. NLTHA are carried out adopting three suites of 10 natural ground motions selected from the European Strong-motion Database (ESD), using the tool REXEL [37] (Figure 5). Such ground motions are linearly-scaled in amplitude to achieve the compatibility with the above-mentioned target spectra (maximum scale factor equal to 5). Spectrum compatibility is ensured in the bandwidth [0.1s, $2 \mathrm{~s}]$, selected to include the values of elastic (secant-to-yielding) first mode period of the analysed bridges, also shown in Figure 5. The observed period shift allows to confirm the appropriateness of the record selection for the time-history analyses. Indeed, all the first mode effective periods (secant-to-performance point) fall within the adopted matching bandwidth.

\section{Results of the parametric analyses}

\subsection{Calculation of the capacity demand ratio and the bridge index}

The analysis results for each case-study bridge are represented by two parameters: the Capacity Demand Ratio (CDR) and the Bridge Index (BI). Those respectively refer to the seismic performance of a given bridge with respect to the demand, and to the accuracy of the predicted displacement profile with respect to the time-history analyses, herein taken as a benchmark. Similarly, the error on the CDR $\left(\frac{N S P-T H}{T H}\right)$ is introduced to have a systematic comparison of the parametric analysis results and the evaluation of DBPP procedure.

For all the considered analysis methods, it is assumed that the first pier that reaches the Ultimate Limit State (ULS) causes the bridge ULS. For the NSPs, referring to pushover or DBPP, the CDR is defined by post-processing the results 
according to Eq. 17. The displacement capacity of each pier $\left(\Delta_{U p i e r, k}\right.$, where $k$ indicates the piers) is compared to the displacement demand at the performance point $\left(\Delta_{p i e r, k}^{P P}\right)$. These refer to the displacement profile consistent with the performance point calculated with the CSM. To calculate the CDR of the time-history analyses for a suite of records (Eq. 18), the displacement capacity of each pier is compared to the average response for the considered ground motion suite $\left(\Delta_{\text {pier }, k}^{T H}\right)$.

It is worth mentioning that, since no strength degradation is considered in this study, an hardening behaviour is registered in the force-displacement curves after the attainment of the ULS, and this also affects the calculation of the performance point for bridges that do not meet the criterion $C D R \geq 1$. Clearly, other members in the bridge (abutments, bearings, etc.) should be considered in the calculation of the CDR. However, this is outside the scope of this paper and only the piers are herein considered.

$$
\begin{aligned}
& C D R^{N S P}=\min \left(\frac{\Delta_{U p i e r, k}}{\Delta_{\text {pier }, k}^{P P}}\right) \\
& C D R^{T H}=\min \left(\frac{\Delta_{U p i e r, k}}{\Delta_{\text {pier }, k}^{T H}}\right)
\end{aligned}
$$

The BI, proposed by Pinho et al., 2007 [7], and adapted by Kohrangi (2015) [38], is herein adopted as an indicator of the bias of the NSPs with respect to the time-history analysis results in terms of deck displacement profile $\left(\Delta_{i}\right)$. In particular, the maximum response of each NLTHA run is used to calculate the equivalent SDoF displacement. The average of such displacements for the considered ground-motion suite is calculated $\left(\Delta_{S D o F}^{T H}\right)$. The corresponding displacement profile $\left(\Delta_{i}^{N S P}\right)$ is extracted from the database of a NSP, and the BI is calculated with Eq. 19, where $N_{\text {sub }}$ is the number of sub-structure members (piers and abutments). Clearly, BI values close to one indicate the accuracy of a NSP in approaching the refined time-history results.

$$
B I=\frac{1}{N_{\text {sub }}} \sum_{i=1}^{N_{\text {sub }}} \frac{\Delta_{i}^{N S P}}{\Delta_{i}^{T H}}
$$

\subsection{Detailed results for selected bridge case studies}

This section presents the detailed results of three selected case studies. This allows to better interpret the overall results presented in Section 4.3 .

The first selected case study is a four-span bridge $(\mathrm{J} 100 \mathrm{~B} 222)$ showing high regularity in the seismic response $\left(R S_{e}=\right.$ 1.26, $R S_{p p}=1.32$ ). Figure 6.a shows the NSP-related curves (PUSHm, PUSHu, DBPPm and DBPPs), representing the effective SDoF displacement versus the total base shear. Both the CSM-based performance points (for the three considered IMs) and the ULS are indicated. Moreover, three indicators show the average response of the time-history analyses (10 runs for each considered suite). In particular, the displacement and shear profiles for each ground motion record are enveloped, before taking their average. An SDoF approximation (displacement and base shear) is derived based on such average profiles. To have a measure of the NLTHA response dispersion, the related confidence ellipses are also shown. Those represent the standard deviation of the effective SDoF displacement and the total base shear, including their correlation.

Figure 6.a indicates that the DBPPs curve is particularly similar to the PUSHm, while the DBPPm predicts a slightly-higher base shear (less than $6 \%$ over-estimation throughout the entire curve). The worst prediction is provided by the PUSHu, for which a $16 \%$ base shear over-estimation at ULS is observed with respect to the PUSHm. In this case, using a uniform force profile introduces a higher shear force directly transferred to the abutments, thus increasing the total base shear. The DBPPs, DBPPm and PUSHm performance points are particularly close to the average NLTHA response, proving the accuracy of the corresponding procedures. For IM3, the error for the displacement is equal to 

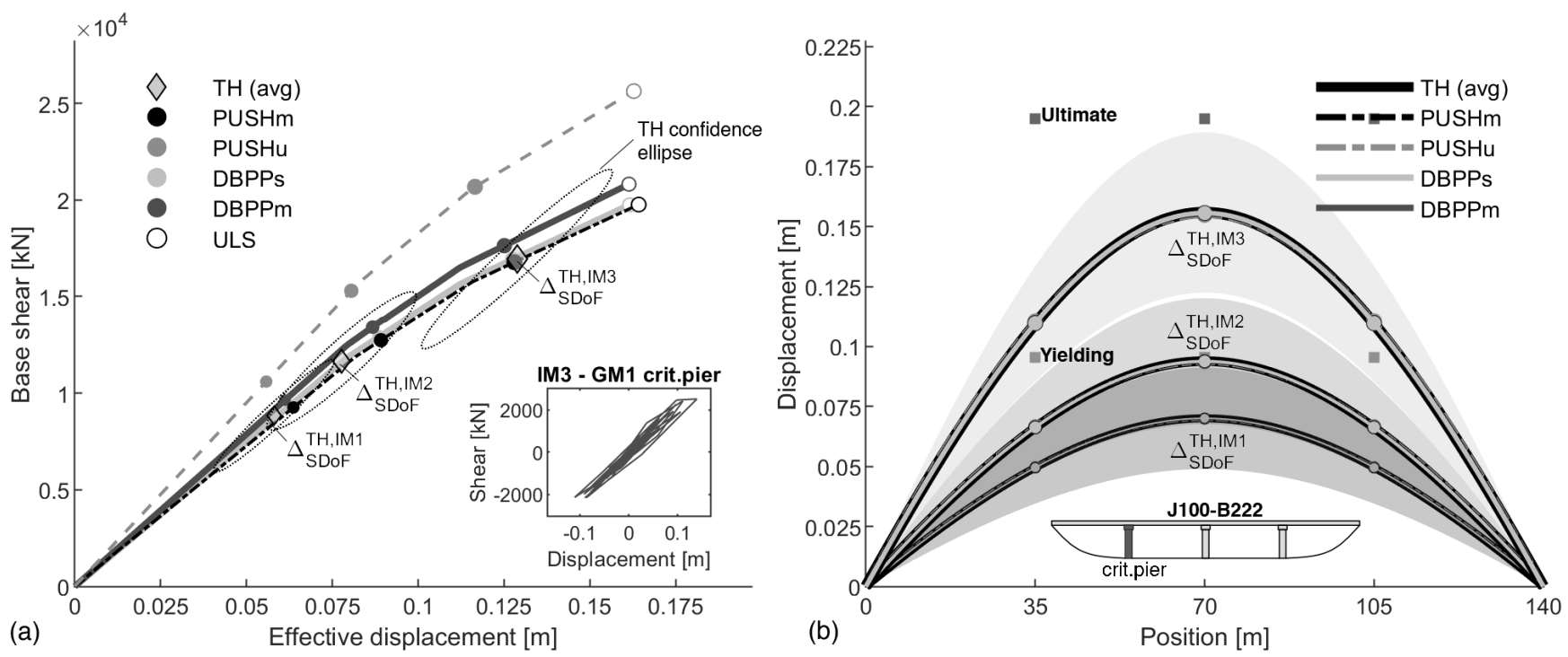

Figure 6: J100 B222 bridge: a) capacity curves and NLTHA response, b) displacement profiles calculated at $\Delta_{S D o F}^{T H}$.

$-1.0 \%,-2.8 \%$ and $-0.5 \%$, respectively for DBPPs, DBPPm and PUSHm $(+8.3 \%,+5.4 \%$ and $+9.7 \%$ for IM1). However, a slightly-higher over-prediction is observed for the IM2 performance-point displacement (respectively $+14.0 \%,+11.6 \%$ and $+14.7 \%$ ). This is possibly related to the damping assumption in the CSM, which may be less accurate in the branch of the capacity curve where the highest stiffness change is registered.

For this case study, the accuracy of the NSPs based on the first modal shape is evident, since the corresponding capacity curves are particularly close to the average NLTHA response for the three analysed IMs, and within the corresponding standard deviation. Moreover, the DBPPs curve is practically identical to the PUSHm one, demonstrating the reliability of the simplified method for first mode-dominated bridges.

The ULS of the system is predicted consistently by using all the NSPs. The CDR based on time-history analysis is equal to 1.25 at IM3, while its relative error with respect to the NLTHA is equal to $+1.1 \%,+3.1 \%$ and $+0.7 \%$, respectively for the DBPPs, DBPPm and PUSHm. Considering the simplicity of the proposed methods with respect to the NLTHA, such error trends are deemed to be satisfactory.

Figure 6.b shows the displacement profiles from the NLTHA (average \pm standard deviation), for each IM. The displacement profiles predicted by each NSP are shown for an SDoF displacement equal to the NLTHA average $\left(\Delta_{S D}^{T H}{ }_{0}\right.$ defined in Section 4.1). It is worth mentioning that a cubic interpolation is adopted herein, to somehow reflect the topology of the elastic deformation of the continuous deck. The yielding and ultimate displacements of each pier are also shown, thus allowing to interpret each stiffness change in the capacity curves. For each IM, the displacement profile of the bridge is effectively captured by all the adopted NSPs (with respect to the NLTHA). This is confirmed by the BI, which is always smaller than 1.001.

The second selected case study is the J50 B12321, whose response is more affected by the piers rather than the deck $\left(R S_{e}=0.05, R S_{p p}=0.11\right)$. Figure 7.a shows that the DBPPs and DBPPm are affected by a negligible error with respect to the PUSHm, while the PUSHu consistently over-estimates the base shear (max $23 \%$ with respect to PUSHm). The CSM performance point is closely matching the average NLTHA response, especially for IM1 and IM3 (e.g. the DBPPs base shear error is respectively equal to $+12.0 \%$ and $-3.0 \%$ ). A higher error is registered for IM2 (15.9\%), although the CSM performance point is within one standard deviation from the NLTHA average. In fact, close to this displacement level, the highest stiffness change is registered in the capacity curve(s), and the record-to-record variability has a higher influence on the NLTHA response (increasing its dispersion). As an example, the yielding of piers 2 and 4 is dependent on the considered ground-motion record (Figure 7.b). Such results are reflected in the calculation of the CDR. At IM3, this is equal to $0.84,0.85,0.87$ and 0.79 , respectively for DBPPs, DBPPm, PUSHm and NLTHA. 

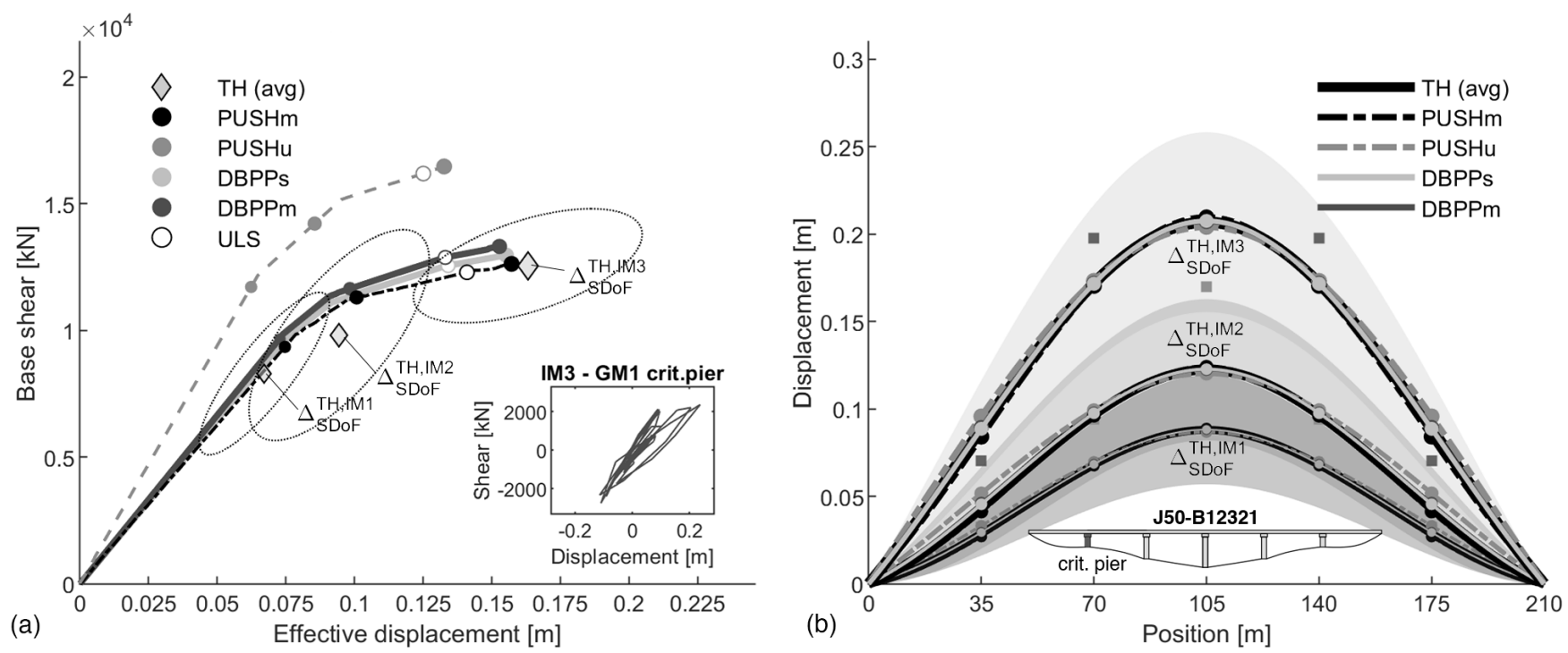

Figure 7: J50 B12321 bridge: a) capacity curves and NLTHA response, b) displacement profiles calculated at $\Delta_{S D o F}^{T H}$. For each pier, a lightand dark-grey markers show their yielding and ultimate displacements.

Figure 7.b shows that the displacement profiles calculated with the NSPs are in good agreement with the average NLTHA results. The BI (IM3) related to the PUSHm is equal to 0.978, while a better performance is observed for the DBPPs and DBPPm (0.999 and 1.002, respectively).

The last selected case study (J100 B1) is a two-span bridge with a 8m-high pier which is clearly dominated by the deck response $\left(R S_{e}=6.44, R S_{p p}=6.44\right)$. It is worth repeating that it is herein chosen to define the ULS of the bridge only according to the piers (not considering bearings, abutments, etc.). For this short bridge, the vast majority of the lateral load is directly carried by the abutments. Therefore, the estimation of the ULS according to the piers (only) leads to particularly high displacement values (outside the limits of the plot in Figure 8.a). Clearly, this is reflected on the CDR.

The results of the analyses show that the bridge remains in the elastic range for all the considered ground motion IMs. The DBPPs is practically coincident to the PUSHm, since both are based on a force profile proportional to the first modal shape. Again, this confirms the reliability of the structural component modelling approach. It is worth mentioning that the DBPPm capacity curve is slightly different from the two previously-mentioned approaches since this is based on a shear profile (rather than force profile) proportional to the first modal shape. Indeed, using the response of the structure rather than the applied forces (shear rather than force profile) allows to better consider the influence of the deck, whose properties affect the shear distribution on the piers/abutments. For this reason, the DBPPs provides equivalent accuracy with respect to the PUSHm and the DBPPm better captures the average NLTHA response (displacement error equal to $-12.9 \%,-6.0 \%$ and $-8.6 \%$, respectively for IM1, IM2 and IM3). The NSPs performance points (for the three IMs) are observed on the plateau of the target spectra. Therefore, the above-mentioned errors are likely caused by the discrepancy between the average ground-motion spectrum and the target one, which is highest in the plateau region (Figure 5).

For this case study, and all the two-span case studies, the BI is equal to 1 regardless of the considered method. Indeed, for such simple bridge configurations, the BI depends solely on the maximum displacement of the pier, and does not provide any added value in the interpretation of the results.

\subsection{Discussion of the results considering the entire dataset}

The results for the entire dataset are discussed herein, including the accuracy of the DBPPs and DBPPm. Considering the NLTHA as a benchmark, Figure 9 shows the error on the CDR while Figure 10 shows the BI. Table 1 shows the CDR values for IM3. Finally, Figure 11 summarises all the conducted analyses. 

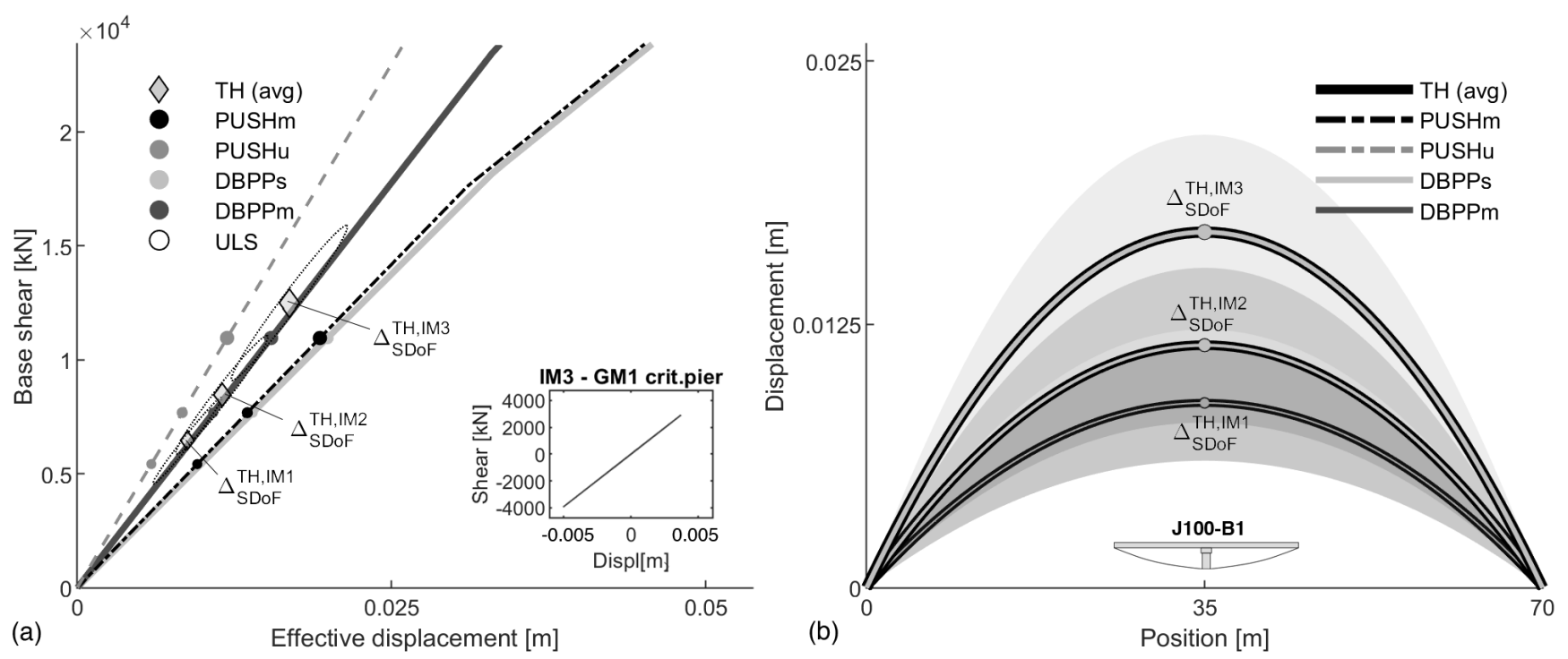

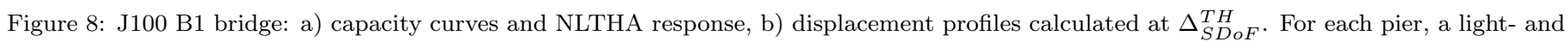
dark-grey markers show their yielding and ultimate displacements.

Considering $R S_{e}$ index, the case-study dataset can be split in three groups. Considering the J50 cases, the two-span bridges $\left(3.24 \leq R S_{e} \leq 38.20\right)$ are dominated by the deck response; for the six-span bridges $\left(0.02 \leq R S_{e} \leq 0.28\right)$, the piers are much more likely to govern the response; the four-span bridges $\left(0.13 \leq R S_{e} \leq 1.59\right)$ represent intermediate cases which can be dominated either by the deck or the piers. Clearly, the $R S_{e}$ for the J100 sub-set are double with respect to the J50 ones. $R S_{p p}=R S_{e}$ for the two-span bridges (elastic response). For the six-span case studies, the small differences between $R S_{p p}$ and $R S_{e}(\Delta R S=0.28$ maximum) indicate that the regularity in their response generally remains unchanged. The response of the four-span case studies is considerably more regular in the inelastic range $(\Delta R S=0.61$ maximum). Indeed, the stiffness degradation for the piers leads to a deck-dominated behaviour.

Figure 9 shows that the DBPPs provides a similar level of accuracy of the DBPPm. In particular, for all the four-spans and six-span case studies, negligible differences in the CDR error are observed. On the other hand, for two-span case studies such differences are higher, indicating a greater accuracy of the DBPPm. This confirms the discussion in Section 4.2, including the higher accuracy of the DBPPm with respect to the PUSHm for two-spans bridges. To validate the assumed equivalent cantilever height in the DBPP approaches, the pier bending moment profiles (PUSHm) are used to provide "numerically-based" estimates of their equivalent height. Repeating the DBPPs using these new height estimates leads to negligible differences in the capacity curve, thus proving the low influence of the deck torsional stiffness.

Considering the four- and six-span case studies, the capacity curve estimation by both the DBPPs and DBPPm closely match the results of the PUSHm (Figure 11). Moreover, the response of all the case studies is dominated by the first vibration mode. The participating mass is always greater than $74 \%$ and therefore the EMS is not necessary (only the first mode is considered). Slightly higher discrepancies are observed for the most irregular configurations in the dataset, i.e. J50 B32123 $\left(R S_{e}=0.08, R S_{p p}=0.25\right)$, J50 B211 $\left(R S_{e}=0.19, R S_{e}=0.39\right)$, J50 B311 $\left(R S_{e}=0.20, R S_{e}=0.81\right)$, where the PUSHm provides the highest base shear, since a non-adaptive approach fails to capture the abrupt stiffness change after the yielding of the shortest piers. The ULS displacement is captured with a relative error (with respect to the PUSHm) equal to $3.3 \%$ and $3.4 \%$ (average of absolute values), respectively for the DBPPs and DBPPm approaches. On the basis of these results, it can be stated that for a wide range of bridge configurations both the DBPPs and DBPPm allow to estimate capacity curves with a level of accuracy particularly similar to a PUSHm.

The CSM is adopted, in conjunction to the DBPPs and DBPPm curves, to assess the seismic performance of the bridges. The resulting performance points are compared to the analogous values obtained with the PUSHm. The relative DBPPs vs PUSHm error is equal to $1.9 \%$ and $2.6 \%$ (average of absolute values considering the three IMs), respectively 
for displacement and base shear. On the other hand, $5.7 \%$ and $2.6 \%$ average errors are respectively registered for the DBPPm. The relative errors with respect to the average NLTHA are equal to $12.4 \%$ and $8.4 \%$ for the DBPPs while $10.6 \%$ and $9.3 \%$ for the DBPPm. This indicates that both methods provide reasonable results when compared to NLTHA analyses, with the DBPPm being slightly better. As shown in Figure 11, for the the majority of the case studies the DBPPm and DBPPs performance points fall within the confidence ellipses of the NLTHA. For IM3, the coefficient of variation of the NLTHA analyses is reported in the range [16\%-33\%] for the effective displacement and [7\%-30\%] for the total base shear.

Figure 9 shows the calculated CDR for each analysis approach. It can be firstly stated that, in estimating the seismic performance, the DBPPs provides a similar accuracy if compared to the PUSHm. By referencing to NLTHA, the average CDR error is equal to $11.0 \%$ and $8.7 \%$ respectively for the DBPPs and the PUSHm. The DBPPm allows for a slightly better accuracy ( $8.2 \%$ average error), since the analyses are based on a shear (rather than force) profile proportional to one or more modal shapes. Finally, the error trends are not sensitive to the moment of inertia of the deck. Indeed, by disaggregating the results for J50 and J100 configurations, a maximum 1\% shift in the above-mentioned average errors is registered. Overall, based on the data in Figure 9, it can be further stated that both the DBPPs and DBPPm approaches provide fairly-accurate seismic performance assessments, if compared to NLTHA analyses.

The PUSHu results greatly over-estimate the base shear capacity for all the case studies (Figure 11). For this reason, the predicted displacement at the performance point is systematically lower than for the PUSHm, DBPPm and DBPPs. With respect to the other NSPs, this causes a higher estimated CDR for the entire dataset, and therefore a higher relative error with respect to NLTHA. In particular, the CDR error falls within the range $[-12.8 \%,+68.3 \%]$. For some case studies (e.g. J50 B121, B211, B311), the PUSHu CDR error is particularly close to zero. However, this does not correspond to a better accuracy of the PUSHu approach. An example is shown in Figure 6 (IM1 and IM2), where the performance point displacement of the PUSHu is particularly similar to the NLTHA one. Although this leads to a low relative error on the $\mathrm{CDR}$, it is clear that the response predicted using the PUSHu is not satisfactory, i.e. the PUSHu performance point falls outside the confidence ellipse of the NLTHA.

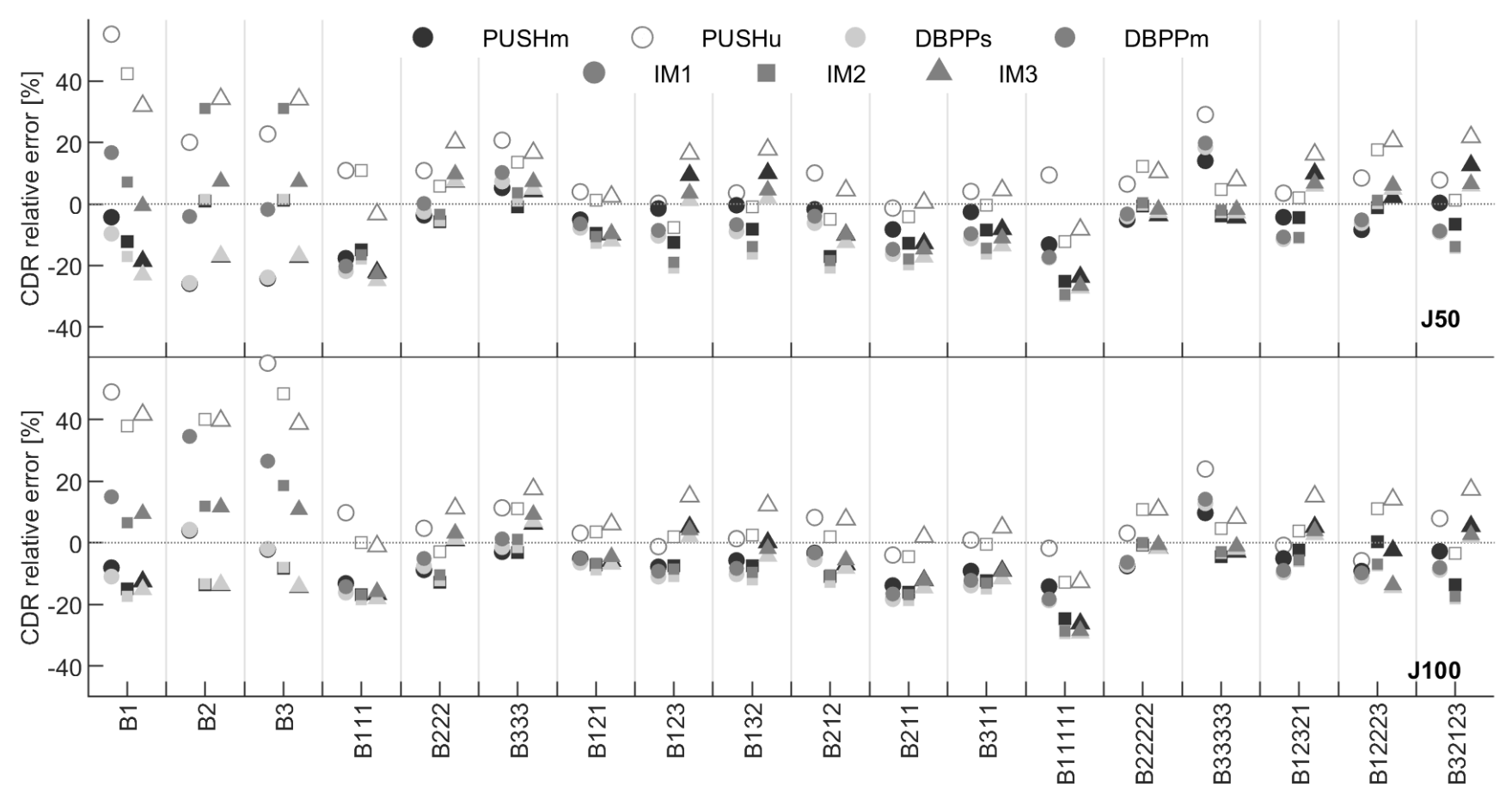

Figure 9: Error of the CDR for the entire dataset relatively to NLTHA.

The accuracy in determining the displacement profile is finally measured calculating the BIs, which are shown in Figure 10.The BIs for the two-span case studies are practically equal to one, and they are not shown in the figure. It 


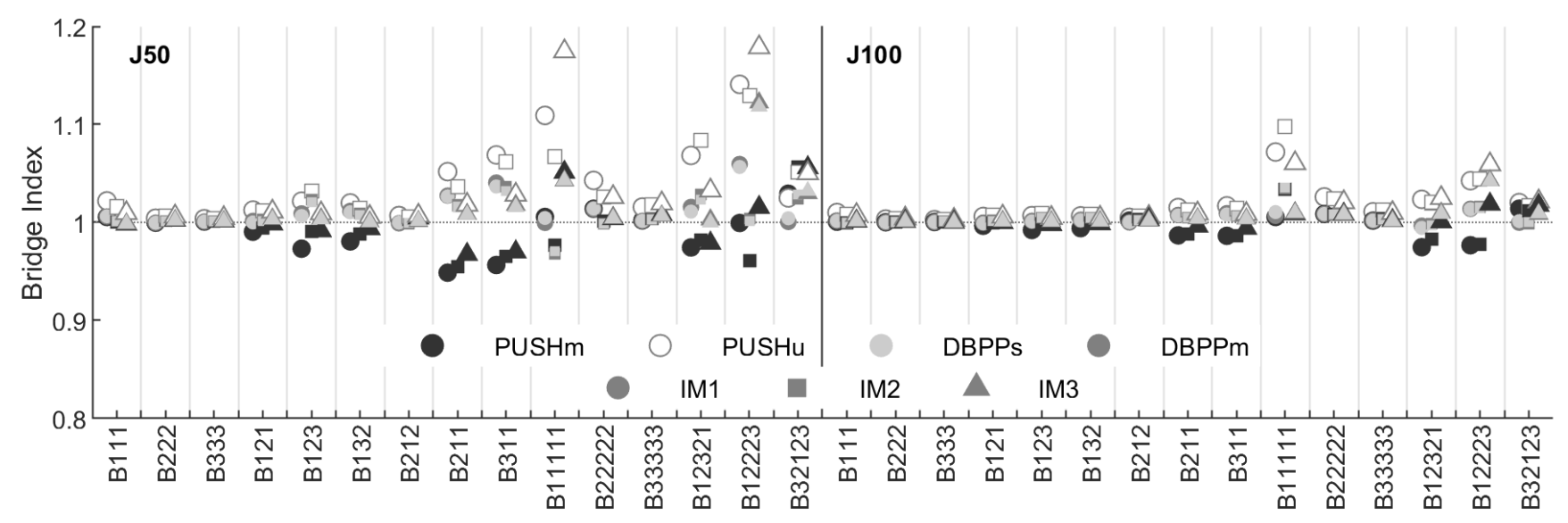

Figure 10: BIs for the entire dataset.

is evident that the DBPPs and DBPPm are equivalently accurate for all the analysed bridges (1.01 average BI for both approaches). For the PUSHm and PUSHu, the observed BIs are generally furthest from 1.00, especially for the less regular bridges (e.g. J50 B211, B311, B12321). This is respectively due to the above-mentioned non-adaptive nature of these approaches and the inadequacy of the uniform force profile. The BIs values are partially governed by the stiffness of the deck, which affects the force redistribution after each stiffness change in the non-linear static response. Indeed, the J100 BIs are considerably closer to 1.00 and they show less dispersion.

Table 1: Capacity Demand Ratio (CDR) for the entire case study dataset, calculated at IM3.

\begin{tabular}{|c|c|c|c|c|c|c|c|c|c|c|}
\hline & \multicolumn{5}{|c|}{$\mathrm{J} 50$} & \multicolumn{5}{|c|}{ J100 } \\
\hline & NLTHA & DBPPs & DBPPm & PUSHm & PUSHu & NLTHA & DBPPs & DBPPm & PUSHm & PUSHu \\
\hline B1 & 3.29 & 2.53 & 3.27 & 2.68 & 4.34 & 4.10 & 3.47 & 4.49 & 3.58 & 5.80 \\
\hline B2 & 6.13 & 5.10 & 6.58 & 5.08 & 8.23 & 9.25 & 8.00 & 10.32 & 7.98 & 12.91 \\
\hline B3 & 10.15 & 8.44 & 10.89 & 8.40 & 13.60 & 15.90 & 13.65 & 17.61 & 13.61 & 22.03 \\
\hline B111 & 1.00 & 0.75 & 0.77 & 0.78 & 0.96 & 0.91 & 0.75 & 0.77 & 0.76 & 0.90 \\
\hline B222 & 1.11 & 1.19 & 1.22 & 1.19 & 1.33 & 1.25 & 1.27 & 1.29 & 1.26 & 1.39 \\
\hline B333 & 1.51 & 1.58 & 1.62 & 1.57 & 1.76 & 1.65 & 1.77 & 1.81 & 1.76 & 1.94 \\
\hline B121 & 1.00 & 0.88 & 0.91 & 0.90 & 1.03 & 0.96 & 0.89 & 0.92 & 0.90 & 1.02 \\
\hline B123 & 0.77 & 0.78 & 0.79 & 0.84 & 0.89 & 0.78 & 0.79 & 0.81 & 0.82 & 0.90 \\
\hline B132 & 0.70 & 0.71 & 0.73 & 0.77 & 0.82 & 0.77 & 0.74 & 0.76 & 0.77 & 0.87 \\
\hline B212 & 0.65 & 0.57 & 0.58 & 0.58 & 0.68 & 0.64 & 0.59 & 0.61 & 0.60 & 0.69 \\
\hline B211 & 0.80 & 0.66 & 0.68 & 0.69 & 0.80 & 0.79 & 0.67 & 0.69 & 0.69 & 0.80 \\
\hline B311 & 0.76 & 0.65 & 0.67 & 0.69 & 0.79 & 0.76 & 0.67 & 0.69 & 0.69 & 0.80 \\
\hline B11111 & 0.85 & 0.62 & 0.62 & 0.65 & 0.78 & 0.81 & 0.58 & 0.58 & 0.60 & 0.71 \\
\hline B22222 & 0.92 & 0.90 & 0.91 & 0.89 & 1.02 & 0.90 & 0.89 & 0.90 & 0.89 & 1.00 \\
\hline B33333 & 1.17 & 1.14 & 1.15 & 1.12 & 1.26 & 1.20 & 1.18 & 1.19 & 1.17 & 1.30 \\
\hline B12321 & 0.79 & 0.84 & 0.85 & 0.87 & 0.92 & 0.74 & 0.76 & 0.77 & 0.78 & 0.85 \\
\hline B12223 & 0.93 & 0.98 & 0.99 & 0.95 & 1.12 & 0.92 & 0.79 & 0.80 & 0.90 & 0.87 \\
\hline B32123 & 0.38 & 0.40 & 0.41 & 0.43 & 0.46 & 0.38 & 0.38 & 0.39 & 0.40 & 0.44 \\
\hline
\end{tabular}



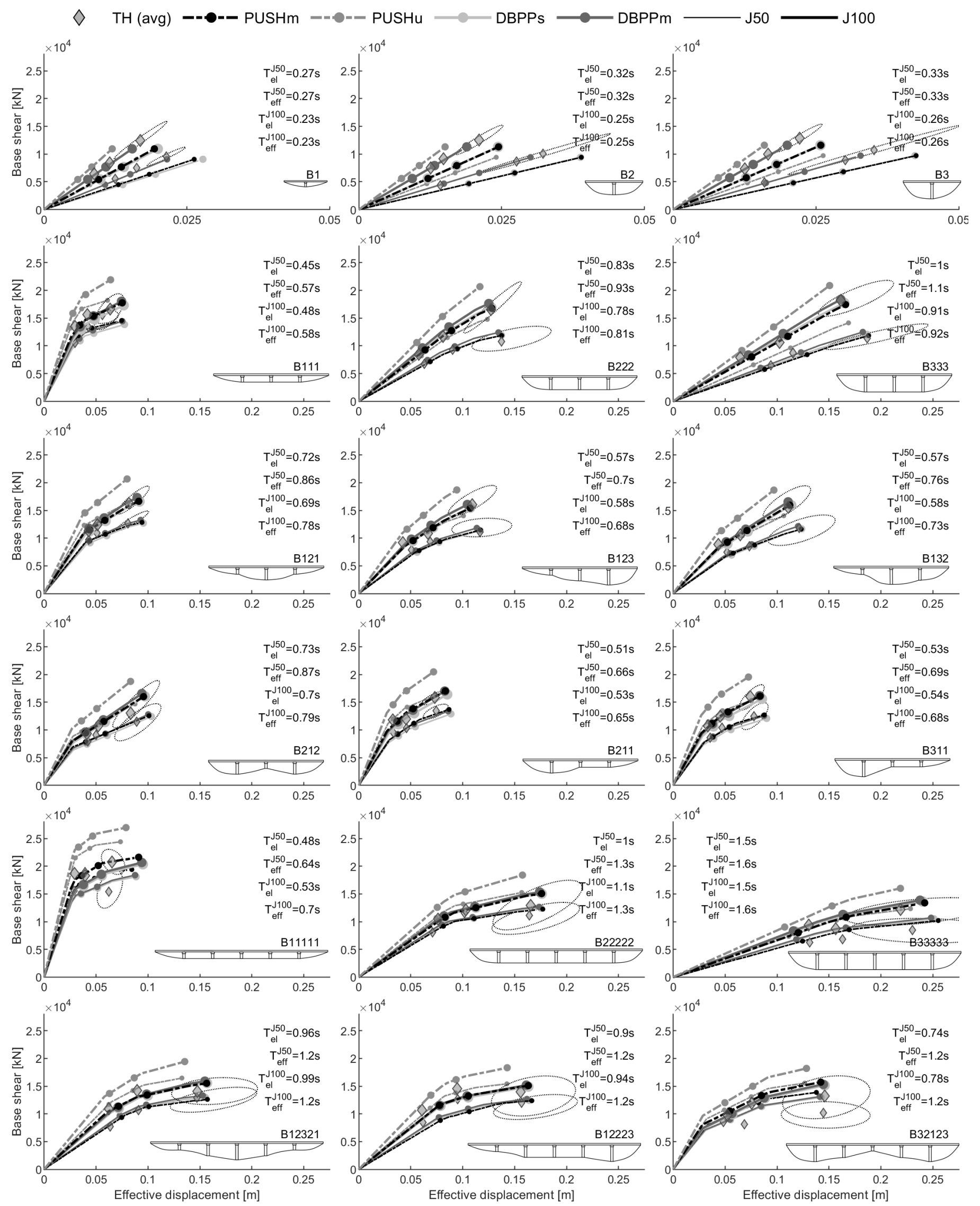

Figure 11: Results of the analyses for the entire case study. The thin curves and the small markers are associated to J50 deck, while the thick curves and the big markers refers to J100. First-mode periods, calculated with elastic (secant-to-yielding) and effective (secant-to-performance displacement, CSM-based) stiffness, are indicated. 


\subsection{Sensitivity analysis for long bridges}

An additional parametric analysis is conducted to investigate the accuracy of the DBPPs and DBPPm for longer bridges ( 8 or more spans). The adopted analysis approaches are the same of the main parametric analysis. The dataset of case studies is composed of six bridges: four having 8 spans and different pier height distribution along the deck (B2222222, B1223221, B3332211, B2131332); two bridges having 10 and 12 spans with 15m-high piers. The J50 deck configuration is provided for all the cases, yielding to $0.01<R S_{e}<0.035$ for the 8-span bridges and $R S_{e}<0.01$ for 10 and 12-span ones. Only the highest seismic intensity is considered (IM3). Given the low $R S_{e}$, the seismic response would be likely governed by the piers. This also increases the sensitivity of higher modes (in both linear and non-linear ranges) to the pier-height distribution.

Figure 12.a investigates the influence of the bridge length only (for a uniform pier-height distribution), by showing the capacity curves of the bridges having 15m-high piers. Figure 12.b, instead, shows the influence of pier-height distribution for the 8-spans cases only (B1223221, B3332211 and B2131332). The DBPPs and PUSHm provide similar capacity curves (as for the shorter bridges), while DBPPm diverges considerably, and tends to the PUSHu. This is caused by the EMS calculated in the DBPPm, since the participating mass of the first mode is smaller than $70 \%$. Confirming the results by Pinho et al., 2007 [7], for long bridge case studies, the NSPs based on the first mode only predict a lower total base shear with respect to the NLTHA. The PUSHu and DBPPm respectively show $-15.1 \%$ and $-16.4 \%$ average relative errors in terms of base shear. Therefore, they respectively outperform the PUSHm and DBPPs, that yield $-38.2 \%$ and $-40.1 \%$ errors. These results are further explained by Figure 13, that reports the displacement profiles at $\Delta_{S D o F}^{T H}$ for the B2131332 and the 12 span-bridge. In the former case, the PUSHu and DBPPm better mimic the TH-based displacement profile, if compared to the first mode-based analysis techniques (PUSHm and DBPPs). Indeed, the PUSHu and DBPPm provide BIs of 1.10 and 0.96, outperforming the PUSHm and DBPPs respectively (BI equal to 0.67 and 0.71). For the 12-span bridge, the DBPPs and PUSHm fail in capturing the displacement profile $(B I<0.67)$, as reported for the 8-span case studies. Even if it considers a combination of vibration modes, the DBPPm is not able to approximate the NLTHA $(B I=0.71)$. A similar condition is registered for the 10 -span case study. Possibly, this relates to the way the significant vibration modes are combined (i.e. SRSS), which may be inappropriate for bridges with 10 spans or more. Further investigations are required to confirm such hypothesis. On the other hand, a better accuracy is given by the PUSHu $(B I=0.92)$, which better mimics the NLTHA response. The CDR for these bridges are generally smaller than 20\%, as reported in Figure 14 in Section 4.5. Although such errors are not excessively high, those mainly refer to the response of the critical pier (on which the CDR is entirely based). Since the accuracy related to the displacement and shear profiles is lower (as demonstrated above), NLTHA is suggested for bridges with 10 spans or more (in conjunction with a non-linear static approach). Based on this sensitivity analysis, the DBPPs and DBPPm procedures are deemed appropriate for the considered bridges up to six spans (approximately $R S_{e}>0.035$ ). For the considered 8-spans case studies (approximately $R S_{e}>0.01$ ), the DBPPm may still be adopted while the DBPPs is inadequate. For bridges with 10 spans or more (approximately $R S_{e}<0.01$ ), NLTHA is suggested. For practical applications, the applicability of the DBPPs and DBPPm may be based on both the number of spans and $R S_{e}$, whichever is most stringent. However, special attention should be given if $R S_{e}$ is particularly close to the indicated threshold of 0.01 .

\subsection{Further sensitivity analyses}

Two sensitivity analyses are conducted to investigate the effectiveness of the displacement-based approaches to: 1) the amount of pier reinforcement; 2) the pier typology. A first dataset of 15 case studies is defined changing the amount of pier longitudinal reinforcement of the J50 B222, B311, B12223, B22222 and B32123 bridges. For each geometrical configuration, the pier reinforcement is set equal to $\rho_{l}=0.35 \%(\mathrm{LR}), \rho_{l}=0.47 \%(\mathrm{MR})$ and $\rho_{l}=0.70 \%$ (HR). Only the highest intensity subset of ground motions (IM3) is considered. Figure 15.a shows the capacity curves of the B222 

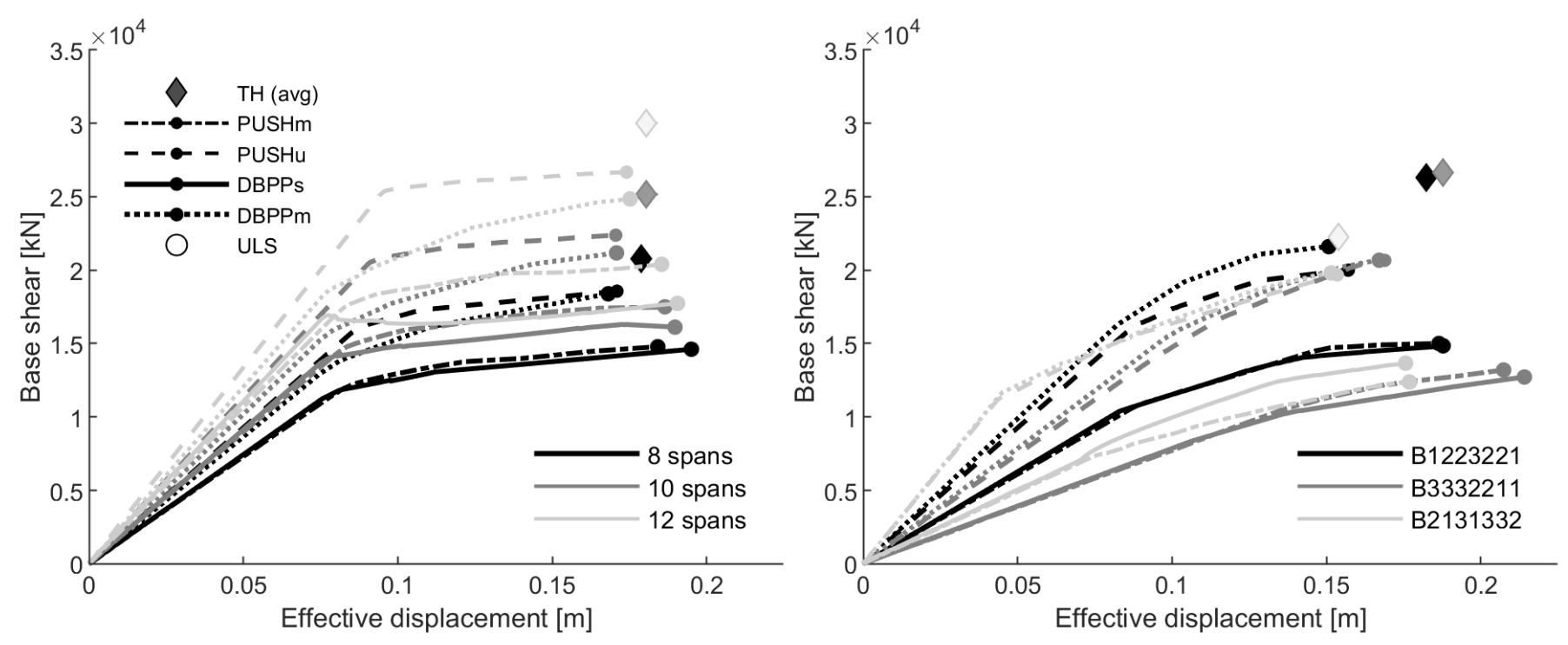

Figure 12: Bridge length sensitivity analysis (capacity curves): a) uniform pier height cases; b) irregular pier height cases.
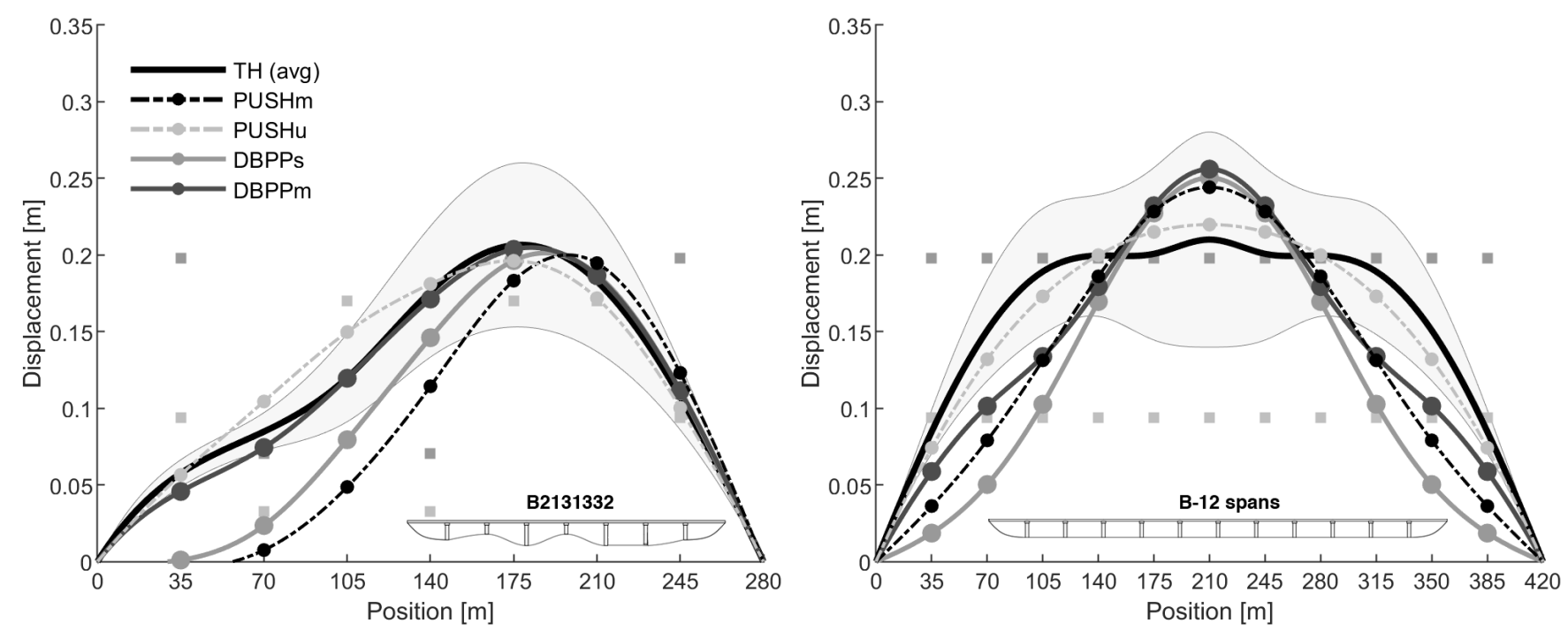

Figure 13: Bridge length sensitivity analysis (displacement profiles): a) B2131322 case study; b) 12-span case study. For each pier, a lightand dark-grey markers show their yielding and ultimate displacements.

bridge, for which the above-mentioned errors (on both displacement and base shear) are in the same order of magnitude, regardless of the pier reinforcement. The relative errors (NSPs vs NLTHA) on the CDRs reported in Figure 14 confirm this outcome. It is evident that the discrepancy of the DBPPs and DBPPm with respect to the NLTHA is not sensitive to the pier longitudinal reinforcement.

For the last sensitivity analysis, three additional case studies are considered, which have six spans and different pier typologies: two-column framed-piers; single-wall piers; and single-column piers with hollow-squared cross section (Figure 15b). The force-displacement capacity of such piers is derived analytically according to SLaMA, and their overall behaviour is flexural. It is worth noting that, shear failures can be likewise considered in the displacement-based procedures by appropriately reducing the displacement capacity of the members and/or modifying their capacity curve accordingly. The PUSHm, DBPPs and DBPPm curves are particularly similar for these case studies, while the PUSHu consistently overestimates the base shear. The capacity curves reported in Figure 15.b prove that the pier typology does not affect the consistency between the NSPs (PUSHm, DBPPs and DBPPm) and the NLTHA, which is instead affected by the stiffness of the piers (and consequently a lower accuracy is expected for bridges with low RS). Consistently with the previously-considered parametric analyses, the relative error on the displacement at the performance point with respect 


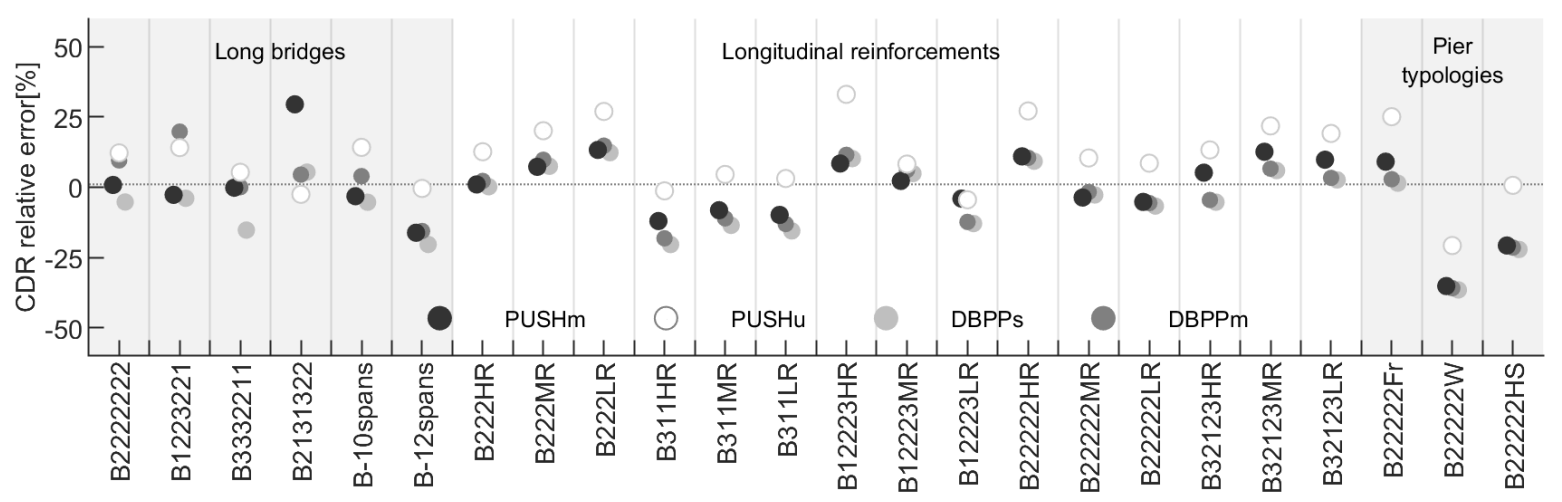

Figure 14: Relative errors on the CDRs for the case studies in the sensitivity analyses.
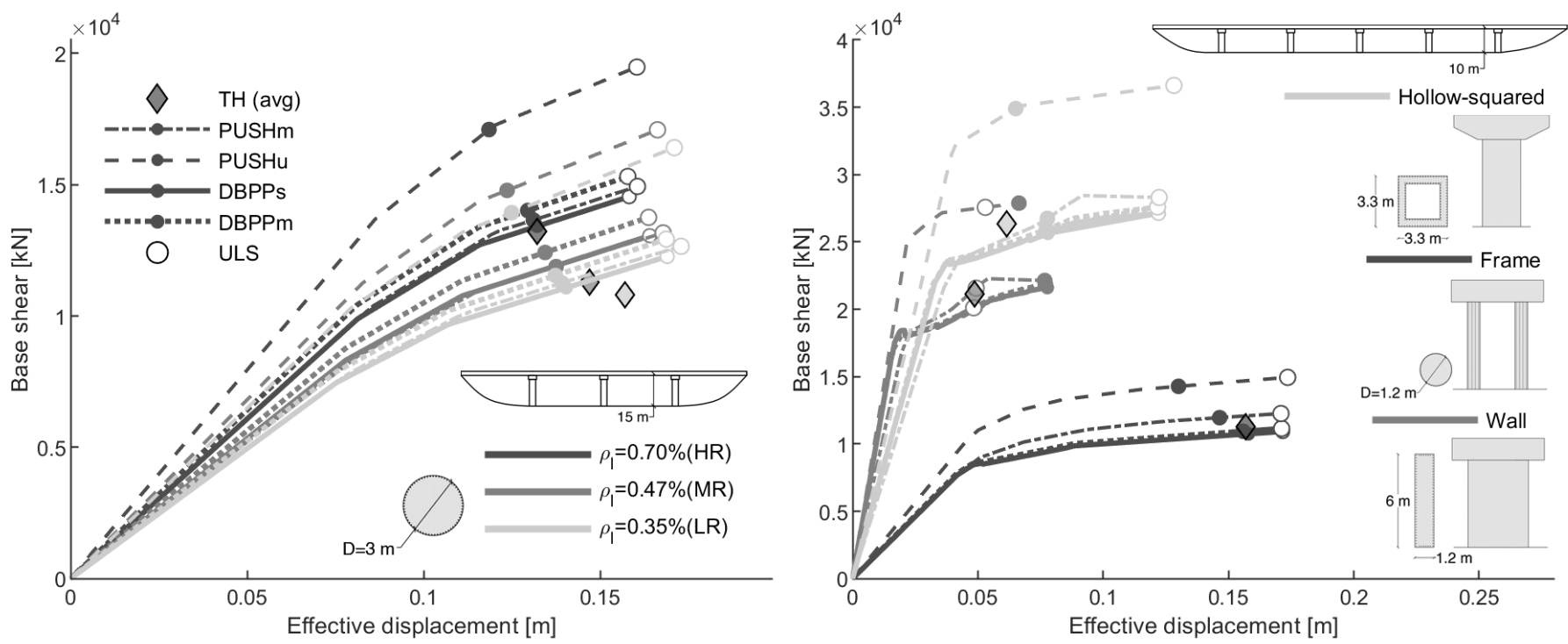

Figure 15: a) sensitivity analysis involving the pier longitudinal reinforcement; b) sensitivity analysis involving the pier typology.

to the NLTHA is higher for the case studies with lower $R S_{e}$. Considering the DBPPm as an example, the relative errors on the CDRs are equal to $+3 \%$ for the framed-pier case $\left(R S_{e}=0.07\right),-21 \%$ for the case study with hollow-squared piers $\left(R S_{e}=0.02\right)$ and $-35 \%$ for the wall-piers bridge $\left(R S_{e}=0.012\right)$. This error trend agrees with the previously-discussed applicability conditions of the DBPP approaches. Indeed, the higher error for wall-piers bridge is expectable, since its $R S_{e}$ value is particularly close to the suggested threshold of 0.01 .

\section{Conclusions}

This paper deals with the seismic performance assessment of continuous-deck RC bridges using DBA. After describing the modal analysis-based DBA procedure, a static-based alternative is proposed in this paper, which is deemed to further increase the simplicity of the DBA approach. Moreover, it is proposed an extension of the DBA procedures, both modal and static, which allows to derive the displacement-based pseudo-pushover curve of the bridge.

The basic idea is to repeat the modal or static DBA procedure for increasing displacements, deriving a thorough information on the behaviour of the analysed bridge with a particularly small increase in computational cost. The process is based on a series of linear analyses based on the secant stiffness of the members compatible with increasing levels of displacement (pseudo non-linear approach). The CSM is finally adopted to assess the bridge performance. The procedures can be performed using electronic worksheets or code routines, using analytical static schemes rather than finite element numerical models. An open-access application is provided for the above calculations. 
The displacement-based pseudo pushover, both modal (DBPPm) and static (DBPPs), are herein adopted for the transverse analysis of a set of 36 reinforced concrete continuous-deck bridges with two, four or six, 35m-long spans, two values of the deck moment of inertia (transverse direction) and different combinations of 8m-, 15m- and 20m-high single column piers. The resulting performance assessments are compared with those calculated by means of pushover (with force profile proportional to the first vibration mode, PUSHm, or uniform, PUSHu) and time-history analyses using three suites of 10 scaled, natural ground motions respectively consistent with low-, medium- and high-seismicity sites. Three additional datasets of (24) bridges are adopted to investigate the accuracy of the DBPPs and DBPPm for: 1) increasing length of the bridge; 2) amount of pier longitudinal reinforcement; 3) different pier typologies. The results can be summarised as follows:

- For the analysed bridge configurations up to six spans, the DBPPs and DBPPm approaches allow estimating the bridge capacity curve with a level of accuracy particularly similar to the PUSHm. On the other hand, the PUSHu provides a systematic and considerable over-estimation of the base shear;

- Both the DBPPs and DBPPm (coupled with the CSM) provide fairly-accurate seismic performance assessments, measured in terms of capacity-demand ratio (CDR), with the modal approach being slightly better. For the vast majority of the cases up to six spans, the performance points fall within one standard deviation from the average of the time history analyses, both in terms of displacement and base shear of the equivalent SDoF system. The DBPPm allows for a better accuracy, since the analyses are based on a shear (rather than force) profile proportional to one or more modal shapes. However, the improvement is substantial for two-span bridges and only slight for the four- and six-span ones. The error trends are not sensitive to the moment of inertia of the deck and to the amount of pier longitudinal reinforcement;

- The applicability of the DBPPs and DBPPm may be based on both the number of spans and the relative stiffness index in the elastic range $\left(R S_{e}\right)$, whichever is most stringent. The DBPPs and DBPPm procedures are deemed appropriate for the considered bridges up to six spans (approximately $R S_{e}>0.035$ ). For the considered 8-spans case studies (approximately $R S_{e}>0.01$ ), the DBPPm may still be adopted while the DBPPs is inadequate. For bridges with 10 spans or more (approximately $R S_{e}<0.01$ ), NLTHA is suggested. However, special attention should be given if the $R S_{e}$ is particularly close to the indicated threshold of 0.01 ;

- Additional pier typologies are considered: two-column framed-piers; single-wall piers; and single-column piers with hollow-squared cross section. The pier typology does not affect the consistency between the NSPs (PUSHm, DBPPs and DBPPm) and the NLTHA;

- The seismic performance assessment of RC continuous bridges in the longitudinal direction can be performed using the same DBPP approaches. Given the much higher simplicity of the involved static scheme (non-linear springs in parallel), numerical validation is not deemed necessary in this particular case. Contrarily, validation is needed for bridge configurations with slider deck-abutment connections (in the transverse direction).

It is finally claimed that the DBPPs and DBPPm can represent a valid alternative to numerical pushover-based approaches to assess the seismic performance of RC continuous bridges with geometrical/mechanical configurations similar to the analysed dataset. Such features render these methods also suitable for the mechanics-based analysis of large bridge portfolios, while containing computational cost.

\section{Acknowledgements}

This study was conducted as part of the Industrial PhD research program (PON-RI 2014-2020) sponsored by the Italian Ministry of Education, University and Research. Additional funding is received by the European Union's Horizon 
2020 research and innovation programme under grant agreement No. 843794. (Marie Skłodowska-Curie Research Grants Scheme MSCA-IF-2018).

\section{References}

[1] A. Palermo, R. Liu, A. Rais, B. Mchaffie, K. Andisheh, S. Pampanin, R. Gentile, I. Nuzzo, M. Graniero, G. Loporcaro, C. Mcgann, L. Wotherspoon, Performance of road bridges during the 14 November 2016 Kaikoura earthquake, Bullettin of the New Zealand Society for Earthquake Engineering 50 (2) (2017) 253-270.

[2] M. J. N. Priestley, F. Seible, G. M. Calvi, Seismic design and retrofit of bridges, John Wiley and Sons, New York, USA, 1996.

[3] A. S. Elnashai, Advanced inelastic static (pushover) analysis for earthquake applications, Structural Engineering and Mechanics 12 (1) (2001) 51-69. doi:10.12989/sem.2001.12.1.051.

[4] B. Gupta, S. K. Kunnath, Adaptive Spectra-Based Pushover Procedure for Seismic Evaluation of Structures, Earthquake Spectra 16 (2) (2000) 367-392. doi:10.1193/1.1586117.

[5] S. Antoniou, R. Pinho, Advantages and limitations of adaptive and non-adaptive force-based pushover procedures, Journal of Earthquake Engineering 8 (4) (2004) 497-522. doi:10.1080/13632460409350498.

[6] S. Antoniou, R. Pinho, Development and verification of a displacement-based adaptive pushover procedure, Journal of Earthquake Engineering 8 (5) (2004) 643-661. doi:10.1080/13632460409350504.

[7] R. Pinho, C. Casarotti, S. Antoniou, A comparison of single-run pushover analysis techniques for seismic assessment of bridges, Earthquake Engineering and Structural Dynamics 36 (10) (2007) 1347-1362. doi:10.1002/eqe.684.

[8] D. Cardone, Displacement limits and performance displacement profiles in support of direct displacementbased seismic assessment of bridges, Earthquake Engineering \& Structural Dynamics 43 (2014) $1239-1263$. doi:10.1002/eqe.2396.

[9] E. Cosenza, C. Galasso, M. Maddaloni, A simplified method for flexural capacity assessment of circular RC crosssections, Engineering Structures 33 (3) (2011) 942-946. doi:10.1016/j.engstruct.2010.12.015.

[10] R. Gentile, F. Porco, D. Raffaele, G. Uva, Simplified Moment-Curvature relationship in analytical form for RC circular sections, Bulletin of the New Zealand Society for Earthquake Engineering 51 (3) (2018) $145-158$.

[11] R. Gentile, D. Raffaele, Simplified analytical Moment-Curvature relationship for hollow circular RC cross-sections, Earthquakes and Structures 15 (4) (2018) 419-429.

[12] M. J. N. Priestley, G. M. Calvi, M. J. Kowalsky, Displacement-based seismic design of structures, IUSS Press, Pavia, Italy, 2007.

[13] M. J. Kowalsky, A displacement-based approach for the seismic design of continuous concrete bridges, Earthquake Engineering \& Structural Dynamics 31 (2002) 719-747. doi:10.1002/eqe.150.

[14] G. Adhikari, L. Petrini, G. M. Calvi, Application of direct displacement based design to long span bridges, Bulletin of Earthquake Engineering 8 (4) (2010) 897-919. doi:10.1007/s10518-010-9173-y.

[15] A. J. Kappos, K. I. Gkatzogias, I. G. Gidaris, Extension of direct displacement-based design methodology for bridges to account for higher mode effects, Earthquake Engineering \& Structural Dynamics (July 2012) (2013) 581-602. doi:10.1002/eqe. 
[16] O. B. Şadan, L. Petrini, G. M. Calvi, Direct displacement-based seismic assessment procedure for multi-span reinforced concrete bridges with single-column piers, Earthquake Engineering \& Structural Dynamics 42 (7) (2013) 1031-1051. doi:10.1002/eqe.

[17] P. Ni, L. Petrini, R. Paolucci, Direct displacement-based assessment with nonlinear soil--structure interaction for multi-span reinforced concrete bridges, Structure and Infrastructure Engineering 10 (9) (2014) 1211-1227. doi:10.1080.

[18] NZSEE, New Zealand Society for Earthquake Engineering, The seismic assessment of existing buildings - technical guidelines for engineering assessments. Wellington, New Zealand (2017).

[19] R. Gentile, C. Del Vecchio, S. Pampanin, D. Raffaele, G. Uva, Refinement and Validation of the Simple Lateral Mechanism Analysis (SLaMA) Procedure for RC Frames, Journal of Earthquake Engineering in press.

[20] R. Gentile, S. Pampanin, D. Raffaele, G. Uva, Non-linear analysis of RC masonry-infilled frames using the SLaMA method: part 1-mechanical interpretation of the infill/frame interaction and formulation of the procedure, Bulletin of Earthquake Engineering 17 (6) (2019) 3283-3304. doi:10.1007/s10518-019-00580-w.

[21] R. Gentile, S. Pampanin, D. Raffaele, G. Uva, Non-linear analysis of RC masonry-infilled frames using the SLaMA method: part 2-parametric analysis and validation of the procedure, Bulletin of Earthquake Engineering 17 (6) (2019) 3305-3326. doi:10.1007/s10518-019-00584-6.

[22] R. Gentile, S. Pampanin, D. Raffaele, G. Uva, Analytical seismic assessment of RC dual wall/frame systems using SLaMA: Proposal and validation, Engineering Structures 188 (2019) 493-505. doi:10.1016/j.engstruct.2019.03.029.

[23] S. A. Freeman, Development and use of capacity spectrum method, in: 6th U.S. National Conf. Earthquake Engng, Seattle, 1998.

[24] L. Montejo, M. J. Kowalsky, Set of codes for the analysis of reinforced concrete members, Tech. rep., North Carolina State University, Raleigh, North Carolina. (2007).

[25] M. P. Berry, M. Eberhard, Practical Performance Model for Bar Buckling, Journal of Structural Engineering 131 (2005) 1060-1070.

[26] M. J. Kowalsky, M. J. N. Priestley, Improved analytical model for shear strength of circular reinforced concrete columns in seismic regions, ACI Structural Journal 97 (2000) 388-396.

[27] R. Gentile, DBA bridges - practical manual. Free internal report and software (www.robertogentile.org, last access: 15/12/2019). Alpha version of the app can be requested at www.robertogentile.org., Tech. rep. (2019).

[28] C. Casarotti, R. Pinho, An adaptive capacity spectrum method for assessment of bridges subjected to earthquake action, Bulletin of Earthquake Engineering 5 (2007) 377-390.

[29] H. Dwairi, M. J. Kowalsky, Implementation of inelastic displacement patterns in direct displacement-based design of continuous bridge structures, Earthquake Spectra 22 (3) (2006) 631-662. doi:10.1193/1.2220577.

[30] J. B. Mander, M. J. N. Priestley, R. Park, Theoretical stress strain model for confined concrete (1988).

[31] D. J. King, M. J. N. Priestley, R. Park, Computer programs for concrete column design, Research Report 86/12, Tech. rep., University of Canterbury, New Zealand, Department of Civil Engineering (1986).

[32] MATLAB, version 9.5.0.944444 (R2018b), The MathWorks Inc., Natick, Massachusetts, 2018. 
[33] A. J. Carr, RUAUMOKO3D - The Maori God of Volcanoes and Earthquakes. Inelastic Analysis Finite Element program., Tech. rep., Carr Research Ltd., Christchurch, New Zealand (2016).

[34] R. D. Sharpe, The Seismic Response of Inelastic Structures, Ph.D. thesis, Department of Civil Engineering, University of Canterbury, Christchurch, New Zealand. (1976).

[35] M. Saiidi, M. Sozen, Simple and complex models for nonlinear seismic response of reinforced concrete structures, Tech. Rep. Report No 465, University of Illinois, Urbana, Illinois, USA (1979).

[36] NTC2018, DM 17 gennaio 2018 in materia di "aggiornamento delle norme tecniche per le costruzioni". Gazzetta ufficiale n.42 del 20 febbraio 2018, Tech. rep. (2018).

[37] I. Iervolino, C. Galasso, E. Cosenza, REXEL: Computer aided record selection for code-based seismic structural analysis, Bulletin of Earthquake Engineering 8 (2) (2010) 339-362. doi:10.1007/s10518-009-9146-1.

[38] M. Kohrangi, R. Bento, M. Lopes, Seismic performance of irregular bridges - comparison of different non linear static procedures, Structure and Infrastructure Engineering 11 (12) (2015) 1632-1650. 